\title{
37. CHEMICAL COMPOSITION OF LEG 138 SEDIMENTS AND HISTORY OF HYDROTHERMAL ACTIVITY ${ }^{1}$
}

\author{
Evgeny G. Gurvich, ${ }^{2}$ Mikhail A. Levitan, ${ }^{2}$ and Tat'yana G. Kuzmina ${ }^{2}$
}

\begin{abstract}
Three main sedimentary sequences are briefly characterized in geochemical terms. Metalliferous sediments have lower average contents of $\mathrm{Fe}, \mathrm{Mn}$, and combined microelements, and higher average contents of $\mathrm{Ti}$ and $\mathrm{Al}$, than other recent and ancient metalliferous sediments of the Pacific Ocean. We propose the history of hydrothermal activity in the eastern equatorial Pacific for the last 17 m.y.
\end{abstract}

\section{INTRODUCTION}

Leg 138 was devoted to the study of late Cenozoic evolution of global climate in the eastern equatorial Pacific Ocean. At the same time, we obtained evidence for hydrothermal activity of the East Pacific Rise (EPR) during the Neogene (Mayer, Pisias, Janecek, et al., 1993), for example, metalliferous sediments and geochemical anomalies in sediment sequence. Such phenomena in the study region were described earlier during DSDP Legs 9, 16, 54, and 85 (Boström, 1973; Leinen and Stakes, 1979; Rosendahl, Hekinian, et al., 1980; Jarvis, 1985). Nevertheless, many questions still remain about the specifics of (1) chemical composition of metalliferous sediments; (2) sources of many trace and minor elements; (3) relationships among spreading rates, the distance from the EPR axis, and geochemistry of sediments; and (4) the role of bottom currents. Here, we try to answer some of these questions.

\section{METHODS}

We analyzed 544 sediment samples from all the Leg 138 sites. Half of these (one sample per section) were analyzed for $\mathrm{C}_{\text {org }}, \mathrm{CaCO}_{3}$, opal, and, sometimes, $\mathrm{P}_{2} \mathrm{O}_{5} . \mathrm{C}_{\text {org }}$ and $\mathrm{CaCO}_{3}$ were determined using a LECO-type gas analyzer AN-7529, with dry combustion in a stream of oxygen (Lyutzarev, 1986). $\mathrm{P}_{2} \mathrm{O}_{5}$ was analyzed using the classical "wet chemistry" method (Ponomarev, 1961).

After $\mathrm{CaCO}_{3}$, biogenic silica is the second major component in most of these sediments. To determine opal contents, we used double leaching with $5 \%$ hot $\mathrm{Na}_{2} \mathrm{CO}_{3}$ for $2 \mathrm{hr}$, and subsequent treatment with $\mathrm{HCl}$ (Ponomarev, 1961). This technique indicates incomplete opal recovery. For sediments analyzed by X-ray fluorescence (XRF), we used Leinen's normative calculation technique for opal determination (1977). Comparison of wet chemistry with XRF results gave opal correction factors of 1.55 for low-carbonate sediments and 1.30 for highcarbonate sediments measured by chemistry.

The other half of the samples (one sample per section) was analyzed using XRF spectroscopy for 21 major and trace elements. To analyze major elements, we used an XRF spectroanalyzer VRA-30 (Germany) and for trace elements, an XRF spectroanalyzer SPARK-1 (Russia). The VRA-30 has an X-ray tube with a W-anode $(40 \mathrm{kV} / 40$ $\mathrm{mA}$ ) gas proportional (DZ) and scintillation (SZ) detector and flat crystal analyzers: ADP, PE, and LiF. The SPARK-1 has an X-ray tube with an Re-anode shoot-through type, proportional detector and crystal analyzers that focus, according to Iogansson.

\footnotetext{
${ }^{1}$ Pisias, N.G., Mayer, L.A., Janecek, T.R., Palmer-Julson, A., and van Andel, T.H. (Eds.), 1995. Proc. ODP, Sci. Results, 138: College Station, TX (Ocean Drilling Program). ${ }^{2}$ P.P. Shirshov Institute of Oceanology, Russian Academy of Sciences, Moscow,
}

The method used to analyze major elements is described in Turanskaya et al. (1976) and Turanskaya and Kuzmina (1978). Tablets for analyses were pressed from ground (up to 300 mesh), ignited $\left(2 \mathrm{hr}, 1030^{\circ} \mathrm{C}\right)$ sediments with dope (about $2 \%$ of the sample's weight). The technique for analyzing minor elements is described in Ivanov and Krasnyuk (1989) and Krasnyuk and Ivanov (1990).

To test the accuracy of our analyses, we used rock standards from the U.S. Geological Survey (BCR-1, G-1, G-2), the Center of Petrographic and Geochemical Researches of France (BR, GR), the Central Geological Institute of Berlin (BM, KH), and the bottom sediment standards of P.P. Shirshov Institute (SDO-1, SDO-2, SDO3 , SDO-6, SDO-7, SDO-8). Errors (in relative percent) for lowcarbonate samples are within the range of $\pm 1.5 \%$ for $\mathrm{SiO}_{2} ; \pm 3 \%$ for $\mathrm{Al}_{2} \mathrm{O}_{3} ; \pm 3.5 \%$ for $\mathrm{CaCO}_{3}$ and $\mathrm{K}_{2} \mathrm{O} ; \pm 4 \%$ for $\mathrm{TiO}_{2}, \mathrm{MnO}$, and $\mathrm{Fe}_{2} \mathrm{O}_{3}$; $\pm 6 \%$ for $\mathrm{MgO}$ and for high-carbonate samples; $\pm 1.7 \%$ for $\mathrm{SiO}_{2} ; \pm 3 \%$ for $\mathrm{CaO}$; and $\pm 8 \%$ to $10 \%$ for other oxides. For trace elements, errors are within the ranges of $\pm 7 \%$ for $\mathrm{Sr}, \pm 3 \%$ for $\mathrm{Fe}, \pm 10 \%$ for $\mathrm{Fe}, \pm 10 \%$ for $\mathrm{Co}, \pm 7 \%$ for $\mathrm{Mn}, \pm 10 \%$ for $\mathrm{Cr}, \pm 10 \%$ for $\mathrm{V}, \pm 10 \%$ for $\mathrm{Ba}, \pm 7 \%$ for $\mathrm{Ti}$, and $\pm 20 \%$ for $\mathrm{Zn}$.

We analyzed natural sediments without washing out salts or dissolving $\mathrm{CaCO}_{3}$. Most of the loss on ignition was $\mathrm{CO}_{2}$ that had been driven off by the carbonate. A strong positive correlation exists between XRF and wet chemistry $\mathrm{CaCO}_{3}$ data; but on average, the wet chemistry value is 1.08 the $\mathrm{XRF}$ value. Determinations for $\mathrm{Al}_{2} \mathrm{O}_{3}$ and $\mathrm{Na}_{2} \mathrm{O}$ were good in low-carbonate sediments, but unsatisfactory in high-carbonate and metalliferous sediments. As a result, the application of Leinen's technique (1977) for determining opal in these sediments required us to calculate the $\mathrm{Al}$ content from the $\mathrm{Ti}$ content, using the $\mathrm{Ti} / \mathrm{Al}$ ratio, which was 0.05 for high-carbonate sediments and 0.07 for metalliferous sediments. Chromium and $\mathrm{P}_{2} \mathrm{O}_{5}$ (XRF) data also were unsatisfactory. The age model for mass accumulation rate (MAR) calculations is presented by Shackleton et al. (this volume). The traditional equation for calculating MARs was presented earlier (Mayer, Pisias, Janecek, et al., 1992).

\section{GEOCHEMICAL CHARACTERISTICS OF THE SEDIMENTARY SEQUENCE}

The lithostratigraphy of each site was described in detail in the Initial Reports volume of Leg 138 (Mayer, Pisias, Janecek, et al., 1992). Outlined below are the brief geochemical characteristics of the sedimentary formations (sequences).

In general, there are three main sedimentary sequences: siliceousclayey (F1), siliceous-carbonate (F2), and a basal metalliferous sequence (F3). The F1 sequence corresponds to Unit I in Site 844 and 845 cross sections. The F3 sediments contain more than $10 \% \mathrm{Fe}$ (OCFM) (opal-carbonate-free matter) (Lisitzin at al., 1990) and have a (Fe + Mn)/Ti ratio of more than 25 (Strakhov, 1976). These sediments occur 


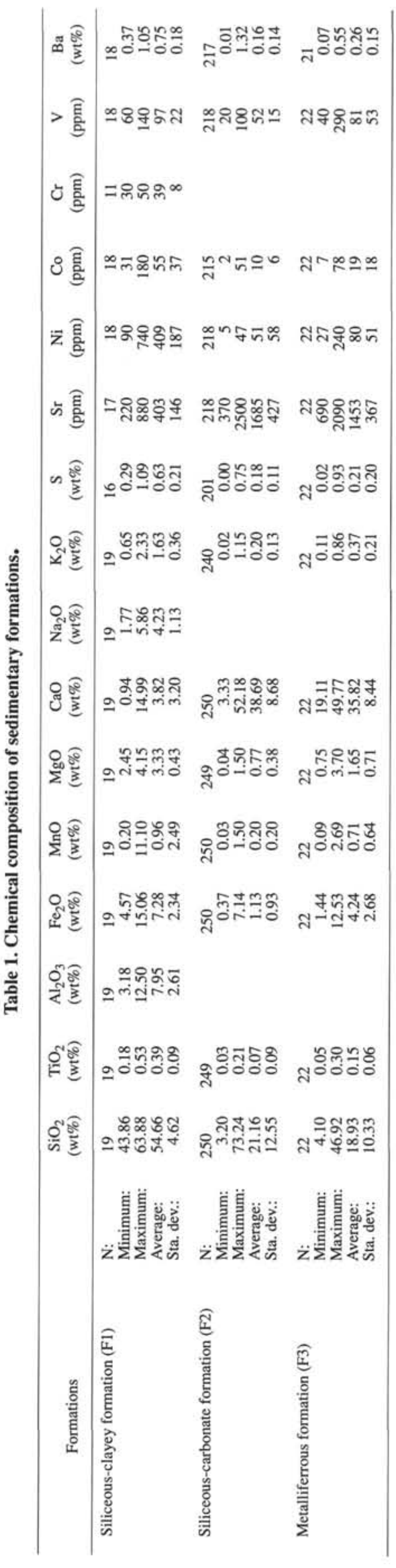

mainly near the base of the sedimentary section above the basalt basement. The F2 sequence coincides with Unit II in Sites 844 and 845 , and with Unit I in all other sites (with the exception of F3 sediments). All the sedimentary sequences have a different stratigraphic thickness in different sites. For our purposes, it is important that F3 sediments are diachronous (from middle to late Miocene in age).

The question of the importance of the diagenetic redistribution of chemical elements comes up. We have much evidence of diagenetic processes in the studied sediments: the brown/green boundary, diagenetic lamination, haloes, and microconcretions, and so forth. Nevertheless, we think that this diagenetic redistribution is of some importance in terms of generalization of chemical data for large sections of sediments.

Sedimentary sequence $\mathrm{F} 1$ is characterized by high contents of $\mathrm{SiO}_{2}, \mathrm{TiO}_{2}$, and other major oxides, except $\mathrm{CaO}$ (Table 1). The sequence also has a high content of minor elements, with the exception of Sr. The chemical composition of F2 and F3 sequences show a strong similarity (Table 1). Obviously, the Fe content, $(\mathrm{Fe}+\mathrm{Mn}) / \mathrm{Ti}$ ratio, and MAR of OCFM are higher in F3 than in F2. In general, F1 has a large abundance of terrigenous matter and biogenic opal; F2 has high content of $\mathrm{CaCO}_{3}$; and $\mathrm{F} 3$ contains much hydrothermal matter. "Wet chemistry" data indicate a positive correlation between $\mathrm{C}_{\text {org }}$ and opal $(R=0.55)$ in $\mathrm{F} 1$ sediments; between $\mathrm{P}_{2} \mathrm{O}_{5}$ and OCFM $(R=0.54)$ in F2 sediments; and a lack of correlation between other components in F1, F2, and F3 sediments.

It is interesting to compare XRF data for the chemical composition of OCFM from different sedimentary sequences (Table 2). The F2 sediments are the poorest in all of the elements analyzed, with the exception of $\mathrm{V}, \mathrm{Cu}$, and $\mathrm{Zn}$. The $\mathrm{F} 1$ sediments are rich in many elements connected with terrigenous matter, and F3 sediments are enriched in Fe, Mn, and V. The differences between F1 and F2 OCFM result from geochemical differentiation in the pelagic direction and to greater eolian influx in F2 sediments.

In general, sediment chemistry is defined by ratios between MARs of $\mathrm{CaCO}_{3}$, opal, and OCFM, and also by the chemical composition of OCFM. We characterized the chemical composition of F2 sediments separately in nannoplankton zones (Table 3), using shipboard stratigraphic data by I. Raffi and H. Flores (Mayer, Pisias, Janecek, et al., 1992). The richest contents of opal (more than $20 \%$ ) were observed in sediments of Zones CN6/CN8 (maximum, CN7), CN13, and $\mathrm{CN} 14 \mathrm{~b}-15$. The highest carbonate contents (more than $70 \%$ ) are found in Zones CN4-CN5b/6, CN10, and CN10c-CN11. In general, the most siliceous sediments are also rich in OCFM.

High values of $\mathrm{Fe}_{2} \mathrm{O}_{3}$ and $\mathrm{MnO}$ in the most ancient sediments of Zone $\mathrm{CN} 3$ reflect their position relative to the basalt basement in the area of the eastern transect. The same is true of Zone CN7 sediments in the area of the western transect.

Chemical composition data of F2 sediments for 1-Ma intervals are shown in Table 4. The most carbonate (more than $80 \% \mathrm{CaCO}_{3}$ ) sediments accumulated from 16 to 15 and 13 to $12 \mathrm{Ma}$; the most siliceous (more than 20\% opal) sediments, 18-17, 11-8, and 2-1 Ma. MAR maxima are as follows: $\mathrm{CaCO}_{3}$ (more than $2.0 \mathrm{~g} / \mathrm{cm}^{2} / \mathrm{k}$.y.), 17-16, 13-12, and 7-4 Ma; opal (more than $0.59 \mathrm{~g} / \mathrm{cm}^{2} / \mathrm{k} . \mathrm{y}$.), 18-17, 11-9, 7-6, and 5-4 Ma; OCFM (more than $0.30 \mathrm{~g} / \mathrm{cm}^{2} / \mathrm{k} . \mathrm{y}$ ), 18-17, 11-9, and 7-3 Ma.

\section{CHEMICAL COMPOSITION OF METALLIFEROUS SEDIMENTS}

Metalliferous sediments of F3 occur in the basal layers at Sites $845,846,848,849,850,852,853$, and 854 (Fig. 1). At Site 851, metalliferous sediments are absent. The chemical composition of F3 samples is shown in Table 1. Average concentrations of chemical elements for the OCFM in these sediments, and in other comparable recent and ancient metalliferous sediments, are shown in Table 5. According to Table 5, F3 sediments have the lowest Fe and Mn concentrations; low $\mathrm{Cu}, \mathrm{Zn}$, and $\mathrm{Ni}$ concentrations; and the highest $\mathrm{Al}$ 
Table 2. Chemical composition of opal-carbonate-free matter from different sedimentary formations (XRF data).

\begin{tabular}{|c|c|c|c|c|c|c|c|c|c|c|c|c|}
\hline & & $\begin{array}{c}\mathrm{Ti} \\
\text { (wt\%) }\end{array}$ & $\underset{(w t \%)}{K}$ & $\underset{(\mathrm{wt} \%)}{\mathrm{Mg}}$ & $\begin{array}{c}\mathrm{Ba} \\
(\mathrm{wt} \%)\end{array}$ & $\underset{(w t \%)}{F e}$ & $\begin{array}{c}\mathrm{Mn} \\
\text { (wt } \%)\end{array}$ & $\begin{array}{c}\mathrm{Ni} \\
(\mathrm{ppm})\end{array}$ & $\begin{array}{c}\text { Co } \\
\text { (ppm) }\end{array}$ & $\begin{array}{c}\mathrm{V} \\
(\mathrm{ppm})\end{array}$ & $\begin{array}{c}\mathrm{Cu} \\
(\mathrm{ppm})\end{array}$ & $\underset{(\mathrm{ppm})}{\mathrm{Zn}}$ \\
\hline F1 & $\begin{array}{l}\text { N: } \\
\text { Average: } \\
\text { Sta. dev.: } \\
\text { Minimum: } \\
\text { Maximum: }\end{array}$ & $\begin{array}{l}19 \\
0.36 \\
0.05 \\
0.23 \\
0.46\end{array}$ & $\begin{array}{l}19 \\
2.06 \\
0.63 \\
0.62 \\
3.40\end{array}$ & $\begin{array}{l}19 \\
3.23 \\
0.49 \\
2.40 \\
3.98\end{array}$ & $\begin{array}{l}18 \\
1.19 \\
0.30 \\
0.58 \\
1.93\end{array}$ & $\begin{array}{r}19 \\
8.31 \\
3.13 \\
4.47 \\
17.02\end{array}$ & $\begin{array}{r}19 \\
1.25 \\
3.27 \\
0.26 \\
14.67\end{array}$ & $\begin{array}{r}18 \\
642 \\
269 \\
191 \\
1200\end{array}$ & $\begin{array}{r}18 \\
88 \\
58 \\
48 \\
292\end{array}$ & $\begin{array}{r}18 \\
156 \\
35 \\
101 \\
223\end{array}$ & $\begin{array}{r}18 \\
467 \\
284 \\
266 \\
1492\end{array}$ & $\begin{array}{r}18 \\
609 \\
205 \\
234 \\
1003\end{array}$ \\
\hline $\mathrm{F} 2$ & $\begin{array}{l}\mathrm{N}: \\
\text { Average: } \\
\text { Sta. dev.: } \\
\text { Minimum: } \\
\text { Maximum: }\end{array}$ & $\begin{array}{r}232 \\
0.34 \\
0.08 \\
0.09 \\
0.61\end{array}$ & $\begin{array}{l}223 \\
0.74 \\
0.32 \\
0 \\
2.24\end{array}$ & $\begin{array}{r}232 \\
2.20 \\
0.85 \\
0.03 \\
6.25\end{array}$ & $\begin{array}{r}192 \\
0.74 \\
0.40 \\
0.05 \\
2.57\end{array}$ & $\begin{array}{r}232 \\
3.43 \\
1.76 \\
1.32 \\
12.80\end{array}$ & $\begin{array}{r}232 \\
0.68 \\
0.42 \\
0.14 \\
2.55\end{array}$ & $\begin{array}{r}193 \\
238 \\
239 \\
24 \\
1785\end{array}$ & $\begin{array}{r}190 \\
50 \\
21 \\
8 \\
149\end{array}$ & $\begin{array}{r}193 \\
258 \\
91 \\
97 \\
617\end{array}$ & $\begin{array}{r}138 \\
359 \\
190 \\
76 \\
1218\end{array}$ & $\begin{array}{r}44 \\
561 \\
323 \\
124 \\
1784\end{array}$ \\
\hline F3 & $\begin{array}{l}\text { N: } \\
\text { Average: } \\
\text { Sta. dev.: } \\
\text { Minimum: } \\
\text { Maximum: }\end{array}$ & $\begin{array}{l}22 \\
0.37 \\
0.07 \\
0.25 \\
0.49\end{array}$ & $\begin{array}{l}22 \\
1.31 \\
0.53 \\
0.56 \\
3.29\end{array}$ & $\begin{array}{r}22 \\
4.66 \\
2.30 \\
1.70 \\
10.99\end{array}$ & $\begin{array}{l}21 \\
1.08 \\
0.46 \\
0.39 \\
2.05\end{array}$ & $\begin{array}{r}22 \\
11.87 \\
3.33 \\
7.37 \\
23.64\end{array}$ & $\begin{array}{l}22 \\
2.07 \\
1.42 \\
0.45 \\
5.71\end{array}$ & $\begin{array}{r}22 \\
332 \\
127 \\
142 \\
654\end{array}$ & $\begin{array}{r}22 \\
76 \\
44 \\
29 \\
178\end{array}$ & $\begin{array}{r}22 \\
361 \\
160 \\
160 \\
781\end{array}$ & $\begin{array}{r}22 \\
439 \\
121 \\
141 \\
631\end{array}$ & $\begin{array}{r}17 \\
438 \\
293 \\
122 \\
1321\end{array}$ \\
\hline
\end{tabular}

and $\mathrm{Ti}$ concentrations, compared to other metalliferous sediments of the Pacific Ocean. The excess of these elements, combined with hydrothermal matter, was estimated, assuming that the total content of an element in metalliferous sediment is the sum of the contents in nonbiogenic and nonmetalliferous pelagic matter $\left(E l_{p}\right)$, biogenic carbonate $\left(E l_{c}\right)$, biogenic silica matter $\left(E l_{s}\right)$, and the excess content $\left(E l_{e}\right)$. Contents of $E l_{p}$ were calculated by the formula,

$$
E l_{p}=\mathrm{Al} \cdot E l_{c l} / \mathrm{Al}_{c l},
$$

where $E l_{c l}$ and $\mathrm{Al}_{c l}=$ average concentrations of the elements and $\mathrm{Al}$ in the clay component of these sediments. $E l_{c}$ and $E l_{s}$ were calculated by multiplying average concentrations of the elements in biogenic carbonate and silica by the proportion of biogenic carbonate and silica in the sediment. The $E l_{\mathrm{c}} / \mathrm{Al}_{c l}$ ratios and element concentrations in biogenic carbonate and silica used in the calculation are shown in Table 6.

We assumed that the excess contents of each of the elements listed in Table 6 (except Ba) are associated with hydrothermal matter. However, barite of nonhydrothermal origin is usually the most important carrier of the excess $\mathrm{Ba}$. Nevertheless, barite is the main carrier of $\mathrm{Ba}$ in metalliferous sediments (Boström et al., 1974; Gurvich et al., 1979).

Because iron and manganese are the main elements of hydrothermal matter in metalliferous sediments, the sum of excess $\mathrm{Fe}$ (i.e., $\mathrm{Fe}_{e}$ ) and excess $\mathrm{Mn}$ (i.e., $\mathrm{Mn}_{e}$ ) reflects the hydrothermal matter content in metalliferous sediments. The sum of the accumulation rates of excess $\mathrm{Fe}+\mathrm{Mn}$ is a measure of hydrothermal MAR. The value of the ratio $E l^{\prime}=E l_{e} /\left(\mathrm{Fe}_{e}+\mathrm{Mn}_{e}\right)$ is a measure of an element's content in hydrothermal matter (except Ba).

For all the samples we studied from $\mathrm{F} 3$ sediments, the ratios $\mathrm{El}^{\prime}$ were calculated (i.e., $\mathrm{Fe}^{\prime}, \mathrm{Mn}^{\prime}, \mathrm{Cu}^{\prime}, \mathrm{Zn}^{\prime}, \mathrm{Ni}^{\prime}, \mathrm{Co}^{\prime}, \mathrm{V}^{\prime}$, and $\mathrm{Ba}^{\prime}$ ), along with the accumulation rates of excess $\mathrm{Fe}+\mathrm{Mn}$. These data were analyzed by factor analysis with Varimax rotation of the factor matrix. Two significant factors explain $85.1 \%$ of the variance. The calculated factor scores are shown in Figure 2. Factor 1 explains $48.5 \%$ of the variance. Only the accumulation rate of excess $\mathrm{Fe}+\mathrm{Mn}$ has a significant positive value. $\mathrm{Cu}^{\prime}, \mathrm{Zn}^{\prime}, \mathrm{Ni}^{\prime}, \mathrm{Co}^{\prime}, \mathrm{V}^{\prime}$, and $\mathrm{Ba}^{\prime}$ have significant negative values. This indicates that higher accumulation rates of hydrothermal matter produce lower concentrations of $\mathrm{Cu}, \mathrm{Zn}, \mathrm{Ni}, \mathrm{Co}$, and $\mathrm{V}$ in this matter, and a lower $\mathrm{Ba}_{e} /\left(\mathrm{Fe}_{\mathrm{e}}+\mathrm{Mn}_{\mathrm{e}}\right)$ ratio and vice versa. This also indicates that seawater has the predominant role as the source of excess $\mathrm{Cu}, \mathrm{Zn}, \mathrm{Ni}, \mathrm{Co}$, and $\mathrm{V}$ in $\mathrm{F} 3$ metalliferous sediments and that the hydrothermal source is minor for these elements and for $\mathrm{Ba}$.

Were there a predominant hydrothermal source of excess microelement, then (1) a higher hydrothermal MAR would result in higher microelement concentration (on the OCFM) in metalliferous sediments and (2) concentrations of trace elements in hydrothermal matter would not be changed (or little changed) with change of hydrothermal MAR.

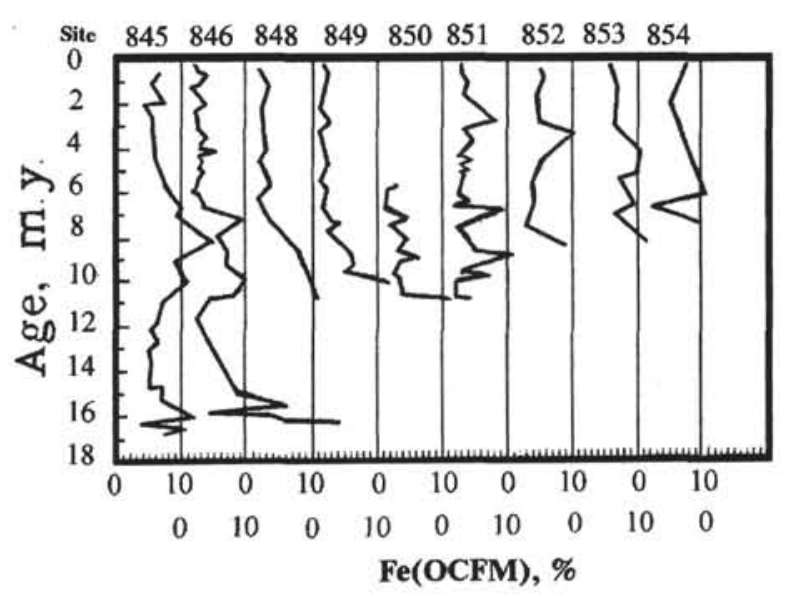

Figure 1. Fe(OCFM) percentages vs. age in sediments from Sites 845,846 , and 848 through 854 .

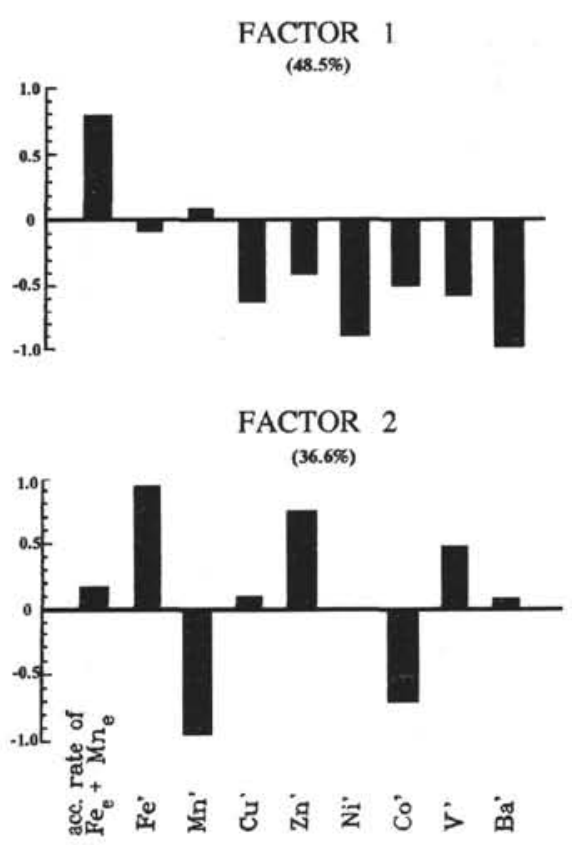

Figure 2. Normalized Varimax factor scores of the parameters in study to two Varimax factors. 
Table 3. Chemical composition of sediments from different biostratigraphic zones of siliceous-carbonate formation (F2; XRF data).

\begin{tabular}{|c|c|c|c|c|c|c|c|c|c|c|c|c|c|c|c|c|}
\hline \multicolumn{17}{|c|}{ Nannoplankton zones } \\
\hline Components & $\mathrm{CN} 3$ & $\mathrm{CN} 4$ & CN5 & $\mathrm{CN} 5 \mathrm{~b} / 6$ & $\mathrm{CN} 6$ & CN7 & CN8 & CN9 & CN10 & $\mathrm{CN} 10 \mathrm{c}-11$ & CNI1 & $\mathrm{CN} 12$ & $\mathrm{CN} 13$ & $\mathrm{CN} 13-14 \mathrm{a}$ & $\mathrm{CN} 14$ & $\mathrm{CN} 14 \mathrm{~b}-15$ \\
\hline $\mathrm{SiO}_{2}$ & 16.46 & 15.59 & 15.15 & 16.42 & 28.76 & 36.86 & 26.10 & 21.48 & 19.40 & 19.95 & 20.00 & 22.91 & 34.14 & 16.23 & 23.73 & 28.69 \\
\hline & (13) 0.13 & 0.12 & 0.11 & $\begin{array}{l}\text { (3) } \\
0.11\end{array}$ & $\begin{array}{l}(8) \\
0.12\end{array}$ & (II) & $\begin{array}{l}(8) \\
0.12\end{array}$ & $\begin{array}{l}\text { (61) } \\
0.12\end{array}$ & 0.10 & (6) 0.11 & ${ }_{0.15}^{(8)}$ & ${ }_{0.23}$ & ${ }_{0.14}^{(8)}$ & 0.15 & 0.19 & $\begin{array}{l}\text { (2) } \\
0.17\end{array}$ \\
\hline$(w+\%)$ & (13) & (11) & (27) & (3) & (8) & (11) & (8) & $(61)$ & (23) & (6) & (8) & (26) & & & & \\
\hline $\mathrm{Fe}_{2} \mathrm{O}_{3}$ & 3.47 & 1.30 & 1.24 & 2.25 & 1.90 & 2.79 & 1.14 & 1.46 & 0.68 & 0.76 & 20 & 1.11 & 1.08 & 155 & & 0.91 \\
\hline & & & (27) ${ }_{022}$ & (3) & ${ }_{0.21}^{(8)}$ & (11) & (8) & (61) & (23) & (6) & (8) & 6) & (8) & (5) ${ }_{034}$ & 17) 029 & (2) \\
\hline (wt\%) & (13) & (111) & (27) & (3) & $\begin{array}{l}0.21 \\
(8)\end{array}$ & (111) & $\begin{array}{l}0.19 \\
(8)\end{array}$ & $(61)^{0.25}$ & (23) & $\begin{array}{l}0.16 \\
(6)\end{array}$ & (8) & (26) & $\begin{array}{l}0.23 \\
(8)\end{array}$ & $\begin{array}{l}0.34 \\
(5)\end{array}$ & $\begin{array}{l}0.29 \\
(17)^{0}\end{array}$ & $\begin{array}{l}0.26 \\
(2)\end{array}$ \\
\hline $\mathrm{MgO}$ & 1.17 & 0.69 & 0.84 & 1.33 & 1.00 & 1.33 & 0.73 & 0.95 & 0.62 & 0.51 & 1.10 & & 0.98 & 066 & 1.28 & 0.79 \\
\hline (wt\%) & (13) & (11) & (27) & (3) & (8) & (11) & (8) & (61) & (23) & (6) & (8) & (25) & (8) & (5) & (17) & (2) \\
\hline $\mathrm{Ca}$ & 38.05 & 42.85 & 42.56 & 41.48 & 33.58 & 25.87 & 37.85 & 38.17 & 40.99 & 40.34 & 37.57 & 36.28 & 28.69 & 38.78 & 34.17 & 31.47 \\
\hline & (13) 0.25 & (11) 0.24 & $(27), 20$ & (3) 0.24 & (8) 0.26 & (11) ${ }_{047}-3$ & (8) & (61) & (23) & (6) & (8) & (26) & (8) & (5) & (17) & (2) \\
\hline $\begin{array}{l}\mathrm{N}_{2} \mathrm{O} \\
(\mathrm{w} \% \%)\end{array}$ & (13) & $\begin{array}{l}0.24 \\
(11)^{2}\end{array}$ & $(27)^{0.20}$ & $\begin{array}{l}0.24 \\
(3)\end{array}$ & $\begin{array}{l}0.26 \\
(8)\end{array}$ & (II) ${ }^{0.47}$ & $\begin{array}{l}0.18 \\
(8)\end{array}$ & $\begin{array}{l}0.28 \\
(53)\end{array}$ & ${ }_{(22)}^{0.13}$ & $\begin{array}{l}0.14 \\
(6)\end{array}$ & ${ }_{(8)}^{0.30}$ & $\begin{array}{l}0.26 \\
(24)\end{array}$ & $\begin{array}{l}0.25 \\
(7)\end{array}$ & $\begin{array}{l}0.2 \\
(5)\end{array}$ & $\begin{array}{l}0.46 \\
(17)^{0.4}\end{array}$ & ${ }_{(2)}^{0.23}$ \\
\hline & 38.02 & 37.02 & 37.44 & 35.56 & 31.63 & 27.72 & - & 34.58 & 36.08 & 36.62 & 37.88 & 35.70 & 31.72 & 39.42 & 32.74 & 34.69 \\
\hline (wt\%) & (13) & (11) & (27) & (3) & (8) & (11) & & $(61)$ & (23) & (6) & (8) & (26) & (8) & (5) & (17) & (2) \\
\hline & 1502 & 1755 & 2022 & 1757 & 1652 & 1351 & 1421 & 1575 & 1717 & 1555 & 1576 & 1566 & 1255 & 1510 & 1460 & 1405 \\
\hline & & & & (3) & (6) & (10) & (8) & (55) & (19) & (2) & (7) & (20) & (9) & (2) & (16) & (2) \\
\hline & 39 & & 22 & 36 & 72 & 68 & 80 & 75 & 41 & 22 & 97 & 71 & 15 & 110 & 120 & 82 \\
\hline & (13) & (11) & (25) & (3) & 6 & & (8) & (55) & (19) & (2) & (7) & (20) & & (2) & (16) & (2) \\
\hline & 8 & & 7 & & 1 & & II & 13 & & & 15 & 15 & i & 2 & 18 & 6 \\
\hline & (13) & (9) & (24) & (3) & $(\theta)$ & (1) & (8) & (55) & 19) & (2) & (7) & & & (2 & (16) & (2) \\
\hline & 83 & 52 & 57 & 60 & 6 & 6 & 60 & 53 & & 40 & 5 & 50 & 53 & 60 & 64 & 65 \\
\hline (ppm) & (13) & (11) & (25) & (3) & (6) & (11) & (8) & $(55$ & (19) & (2) & (7) & (20) & (9) & (2) & (16) & (2) \\
\hline $\mathrm{Ba}$ & 0.14 & 0.15 & 0.15 & 0.12 & 0.32 & 0.37 & 0.17 & 0.18 & 0.31 & 0.06 & 0.26 & 0.17 & 0.19 & 0.34 & 0.25 & 0.22 \\
\hline & & & (25) & (3) & & & (8) & & (19) & (2) & (7) & (20) & (9) & (2) & (16) & (2) \\
\hline Z & 95 & & 70 & - & 125 & & 155 & & 62 & - & 110 & 170 & 19 & 85 & 234 & - \\
\hline & (6) & & (1) & & & ( & ( & & & & & (7) & & (2) & (8) & \\
\hline & 75 & 5 & & 47 & & & & & & 80 & & $\varepsilon$ & & 145 & 99 & - \\
\hline (pp & (13) & (6) & (15) & (3) & (6) & (11) & (8) & (46) & (13) & (2) & (4) & (14) & (6) & (2) & (13) & \\
\hline & & 0.10 & 0.13 & 0.14 & 0.14 & 0.19 & 0.20 & 0.21 & 0.14 & 0.1 & 0.24 & 0.28 & 0.36 & 0.21 & 0.27 & 0.24 \\
\hline & (10) & (5) & (16) & (2) & & (10) & (8) & (50) & & (6) & & (21) & (8) & (5) & (17) & (2) \\
\hline $\mathrm{Mn}$ & 7.37 & 6.32 & 6.14 & 8.49 & 8.24 & 7.24 & 6.34 & 5.56 & 5.55 & 5.76 & 6.97 & 5.89 & 5.96 & 5.08 & 7.50 & 3.19 \\
\hline $\mathrm{SiO}_{2}$ biog & 13.65 & (II) & $\begin{array}{l}(27) \\
12.69\end{array}$ & $\begin{array}{l}\text { (3) } \\
13.26\end{array}$ & $\begin{array}{l}(8) \\
24.37\end{array}$ & $\begin{array}{l}\text { (11) } \\
29.62\end{array}$ & $\begin{array}{l}(8) \\
22.83\end{array}$ & $\begin{array}{l}(63) \\
17.00\end{array}$ & $\begin{array}{l}(24) \\
16.94\end{array}$ & $\begin{array}{l}\text { (6) } \\
17.54\end{array}$ & $\begin{array}{l}\text { (8) } \\
14.93\end{array}$ & $\begin{array}{l}(27) \\
18.96\end{array}$ & $\begin{array}{l}\text { (9) } \\
28.48\end{array}$ & $\begin{array}{l}(5) \\
12.06\end{array}$ & $\begin{array}{l}(18) \\
14.89\end{array}$ & $\begin{array}{l}(22) \\
24.68\end{array}$ \\
\hline & (13) & (11) & (27) & (3) & (8) & (11) & (8) & (61) & $(23)$ & $\begin{array}{l}\text { (6) } \\
\text { lit. }\end{array}$ & $\begin{array}{l}\text { (8) } \\
\text { las }\end{array}$ & (26) & $\begin{array}{l}\text { (8) } \\
\text { (1) }\end{array}$ & (5) & 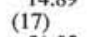 & (2) \\
\hline $\begin{array}{l}\mathrm{CaCO}_{3} \\
(\mathrm{w} \% \%)\end{array}$ & $\begin{array}{l}67.95 \\
(13)\end{array}$ & (11).52 & 76.00 & 74.06 & 59.96 & 46.20 & 67.59 & 68.16 & 76.68 & 72.03 & 67.08 & 64.78 & 51.23 & 69.26 & 51.02 & $\begin{array}{l}56.19 \\
(2)\end{array}$ \\
\hline CFM & 19.28 & 9.89 & 11.49 & 12.96 & 15.37 & 24.36 & 8.71 & 15.07 & 9.53 & 10.10 & 18.74 & 16.46 & & 19.72 & 25.50 & 19.16 \\
\hline & & (11) & (27) & (3) & & (11) & & (61) & (23) & (6) & & (26) & & & & \\
\hline $\mathrm{Fe}+\mathrm{Mn}$ & $\begin{array}{l}33.52 \\
(13)\end{array}$ & 14.37 & 13.93 & 29.40 & 21.01 & 26.94 & 12.77 & 14.25 & 9.55 & 9.82 & 17.94 & 12.71 & 12.54 & 13.63 & 12.42 & 8.59 \\
\hline $\begin{array}{c}\text { In } \\
\text { Bulk } \\
\text { MAR }\end{array}$ & 3.203 & 1.930 & $\begin{array}{l}(27) \\
2.529\end{array}$ & 3.800 & (8) 2.433 & (11) 1.527 & 1.960 & $\begin{array}{l}\text { (63) } \\
2.809\end{array}$ & $\begin{array}{l}\text { (24) } \\
4.387\end{array}$ & $\begin{array}{l}\text { (b) } \\
3.382\end{array}$ & $\begin{array}{l}\text { (8) } \\
1.427\end{array}$ & ${ }_{1.516}^{(27)}$ & 1.541 & (5) 1.059 & ${ }_{1.453}^{(18)}$ & (2) 1.530 \\
\hline$\underset{\left(\mathrm{g} / \mathrm{cm}^{2} /\right.}{\mathrm{MAB}}$ & & & & & & & & & & & & & & & & \\
\hline $\begin{array}{c}\mathrm{MAR} \\
\mathrm{CaCO}_{3} \\
\left(\mathrm{~g} / \mathrm{cm}^{2} / \mathrm{k} . \mathrm{y}\right)\end{array}$ & ${ }_{(8)}^{2.157}$ & ${ }^{111)^{1.478}}$ & ${ }^{126)^{1.918}}$ & ${ }^{2.807}$ & $\begin{array}{l}1.463 \\
(7)\end{array}$ & $\begin{array}{l}0.704 \\
(11)^{2}\end{array}$ & $\begin{array}{l}1.336 \\
(8)\end{array}$ & $\begin{array}{l}1.910 \\
(60)\end{array}$ & ${ }_{(23)^{3.261}}$ & $\begin{array}{l}2.444 \\
(6)\end{array}$ & $\begin{array}{l}0.950 \\
(8)\end{array}$ & $\begin{array}{l}0.980 \\
(26)\end{array}$ & $\begin{array}{l}0.791 \\
(8)\end{array}$ & $\begin{array}{l}0.726 \\
(5)\end{array}$ & $\begin{array}{l}0.874 \\
\text { (17) }\end{array}$ & $\begin{array}{l}0.859 \\
(2)\end{array}$ \\
\hline aR & $\begin{array}{l}0.433 \\
(8)\end{array}$ & ${ }^{0.261}$ & $\begin{array}{l}0.320 \\
(26)\end{array}$ & $\begin{array}{l}0.502 \\
\text { (3) }\end{array}$ & $\begin{array}{l}0.594 \\
(7)\end{array}$ & $\begin{array}{l}0.451 \\
\text { (111) }\end{array}$ & $\begin{array}{l}0.451 \\
(8)\end{array}$ & $\begin{array}{l}0.476 \\
(60)\end{array}$ & ${ }_{(23)^{0.720}}$ & $\begin{array}{l}0.595 \\
(6)\end{array}$ & $\begin{array}{l}0.211 \\
(8)\end{array}$ & $\begin{array}{l}0.286 \\
(26)\end{array}$ & $\begin{array}{l}0.439 \\
(8)\end{array}$ & $\begin{array}{l}0.126 \\
(5)\end{array}$ & $\begin{array}{l}0.213 \\
(17)^{2}\end{array}$ & $\begin{array}{l}0.377 \\
(2)\end{array}$ \\
\hline $\begin{array}{l}\text { MAR } \\
\text { OCFM }\end{array}$ & $\begin{array}{l}0.612 \\
(8)\end{array}$ & $\begin{array}{l}0.191 \\
(11)\end{array}$ & $\begin{array}{l}0.290 \\
(26)\end{array}$ & $\begin{array}{l}0.491 \\
(3)\end{array}$ & $\begin{array}{l}0.375 \\
(7)\end{array}$ & ${ }^{0.371}$ & $\begin{array}{l}0.172 \\
(8)\end{array}$ & $\begin{array}{l}0.422 \\
(60)\end{array}$ & $\begin{array}{l}0.405 \\
(23)^{0}\end{array}$ & $\begin{array}{l}0.342 \\
(6)\end{array}$ & $\begin{array}{l}0.265 \\
(8)\end{array}$ & $\begin{array}{l}0.249 \\
(26)\end{array}$ & $\begin{array}{l}0.311 \\
(8)\end{array}$ & $\begin{array}{l}0.206 \\
(5)\end{array}$ & $\begin{array}{l}0.365 \\
\text { (7) }\end{array}$ & $\begin{array}{l}0.293 \\
(2)\end{array}$ \\
\hline $\begin{array}{l}\text { MAR } \\
\mathrm{Ba}\end{array}$ & $\begin{array}{l}0.003 \\
(8)\end{array}$ & $\begin{array}{l}0.003 \\
\text { (11) }\end{array}$ & $\begin{array}{l}0.003 \\
(25)^{2}\end{array}$ & $\begin{array}{l}0.004 \\
(3)\end{array}$ & $\begin{array}{c}0.003 \\
(7)\end{array}$ & $\begin{array}{l}0.002 \\
\text { (11) }\end{array}$ & $\begin{array}{l}0.002 \\
(8)\end{array}$ & $\begin{array}{l}0.002 \\
(49)\end{array}$ & $\begin{array}{l}0.005 \\
(19)\end{array}$ & $\begin{array}{l}0.003 \\
(2)\end{array}$ & $\begin{array}{l}0.002 \\
(7)\end{array}$ & $\begin{array}{l}0.003 \\
(19)\end{array}$ & $\begin{array}{l}0.003 \\
(9)\end{array}$ & $\begin{array}{l}0.002 \\
(2)\end{array}$ & $\begin{array}{l}0.001 \\
(16)\end{array}$ & ${ }_{(2)}^{0.003}$ \\
\hline $\begin{array}{c}\left(\mathrm{g} / \mathrm{cm}^{2} / \mathrm{k} . \mathrm{y} .\right) \\
\mathrm{MAR} \\
\mathrm{Fe} \\
\left(\mathrm{g} / \mathrm{cm}^{2} / \mathrm{k} . \mathrm{y} .\right)\end{array}$ & $\begin{array}{l}0.039 \\
(8)\end{array}$ & ${ }_{(11)}^{0.014}$ & $\begin{array}{l}0.015 \\
(26)\end{array}$ & $\begin{array}{l}0.056 \\
(3)\end{array}$ & $\begin{array}{l}0.024 \\
(7)\end{array}$ & $\begin{array}{l}0.016 \\
\text { (11) }\end{array}$ & $\begin{array}{l}0.011 \\
(8)\end{array}$ & $\begin{array}{l}0.005 \\
(6)\end{array}$ & $\begin{array}{l}0.019 \\
(24)\end{array}$ & $\begin{array}{l}0.015 \\
(6)\end{array}$ & ${ }_{(8)}^{0.015}$ & $\begin{array}{l}0.011 \\
(27)\end{array}$ & $\begin{array}{l}0.012 \\
(9)\end{array}$ & $\begin{array}{l}0.009 \\
(5)\end{array}$ & $\begin{array}{c}0.011 \\
(18)\end{array}$ & $\begin{array}{l}0.009 \\
(2)\end{array}$ \\
\hline
\end{tabular}

Note: $\mathrm{LOI}=$ loss on ignition.

Previous investigations (Gurvich et al., 1977, 1980a, 1980b; Migdisov et al., 1979) have shown that the enrichment of trace elements in the hydrothermal component of metalliferous sediments as a function of decreasing hydrothermal MAR is evidence that seawater is the main source for these elements in hydrothermal matter. We explain this relationship as follows. There are two reasons for a decrease in hydrothermal MAR:

1. Increase in distance from the hydrothermal source (usually from the crest of a spreading ridge; Rea and Leinen, 1986; Lyle et al., 1987; Lisitzin et al., 1990).

2. Decrease in hydrothermal activity. Increase in distance from a hydrothermal source results in (1) longer contact of suspended hydrothermal particles with seawater and their enrichment in certain trace elements because of their sorption from seawater; (2) longer contact of hydrothermal particles with seawater at the sediment surface because of a decrease in total sedimentation rate. A decrease in total sedimentation rate is the result of (a) decrease of hydrothermal MAR and (b) decrease of $\mathrm{CaCO}_{3}$ accumulation rate. Provided that there is no redeposition of sedimentary matter on a spreading ridge, increased distance from the ridge crest is accompanied by lower $\mathrm{CaCO}_{3}$ accumulation rate because of the greater depositional depth (Bogdanov et al., 1980). Decrease of hydrothermal activity also results in longer contact of hydrothermal matter with seawater at the sediment surface.

One further reason for metal enrichment in slowly accumulating hydrothermal matter is the low concentrations of the metals in seawater, because these low concentrations do not allow the matter to fill to the sorption capacity of hydrothermal matter. During sorption of microelements by hydrothermal matter from equal volumes of seawater, the less flux and accumulation rate of hydrothermal matter, the higher enrichment of hydrothermal matter in microelements (Gurvich et al., 1977, 1980a, 1980b)

The decrease of $\mathrm{Ba}_{e} /\left(\mathrm{Fe}_{e}+\mathrm{Mn}_{e}\right)$, with an increase of hydrothermal MAR, has another cause. The F3 sediments accumulated mainly beneath the high productivity zone, where both metalliferous and 
Table 4. Chemical composition of F2 sediments, generalized on 1-Ma intervals (XRF data).

\begin{tabular}{|c|c|c|c|c|c|c|c|c|c|c|c|c|c|c|c|c|c|c|}
\hline Components & $\begin{array}{c}18-17 \\
\mathrm{Ma}\end{array}$ & $\begin{array}{c}17-16 \\
\mathrm{Ma}\end{array}$ & $\begin{array}{c}16-15 \\
\text { Ma }\end{array}$ & $\begin{array}{c}15-14 \\
\mathrm{Ma}\end{array}$ & $\begin{array}{c}14-13 \\
\mathrm{Ma}\end{array}$ & $\begin{array}{c}13-12 \\
\mathrm{Ma}\end{array}$ & $\begin{array}{c}12-11 \\
\mathrm{Ma}\end{array}$ & $\begin{array}{c}11-10 \\
\mathrm{Ma}\end{array}$ & $\begin{array}{l}10-9 \\
\mathrm{Ma}\end{array}$ & $\begin{array}{l}9-8 \\
\mathrm{Ma}\end{array}$ & $\begin{array}{l}8-7 \\
\mathrm{Ma}\end{array}$ & $\begin{array}{l}7-6 \\
\mathrm{Ma}\end{array}$ & $\begin{array}{l}6-5 \\
\mathrm{Ma}\end{array}$ & $\begin{array}{l}5-4 \\
\mathrm{Ma}\end{array}$ & $\begin{array}{l}4-3 \\
\mathrm{Ma}\end{array}$ & $\begin{array}{l}3-2 \\
\mathrm{Ma}\end{array}$ & $\begin{array}{l}2-1 \\
\mathrm{Ma}\end{array}$ & $\begin{array}{l}1-0 \\
\mathrm{Ma}\end{array}$ \\
\hline $\mathrm{SiO}_{2}$ & 28.47 & 14.44 & 9.47 & 17.69 & 14.44 & 12.41 & 15.32 & 27.99 & 34.06 & 27.78 & 18.57 & 20.47 & 17.97 & 19.70 & 20.99 & 19.59 & 28.96 & 17.83 \\
\hline (wt\%) & (3) & (4) & (3) & (5) & (7) & (5) & (8) & (15) & (16) & (9) & (14) & (28) & (29) & & (18) & & & \\
\hline $\begin{array}{l}\mathrm{K}_{2} \mathrm{O} \\
\left(\mathrm{w}_{1} \%\right)\end{array}$ & 0.17 & $\begin{array}{c}0.18 \\
(4)\end{array}$ & 0.23 & $\begin{array}{l}0.19 \\
(5)\end{array}$ & 0.20 & $\begin{array}{l}0.22 \\
(5)\end{array}$ & $\begin{array}{l}0.18 \\
(8)\end{array}$ & $\begin{array}{l}0.17 \\
(15)\end{array}$ & $\begin{array}{r}0.27 \\
(16)\end{array}$ & 0.22 & $\begin{array}{r}0.15 \\
(14)^{2}\end{array}$ & 0.17 & $\begin{array}{l}0.14 \\
(26)\end{array}$ & $\begin{array}{l}0.13 \\
(30)\end{array}$ & $\begin{array}{r}0.27 \\
(16)\end{array}$ & 0.24 & 0.25 & 0.26 \\
\hline $\mathrm{Sr}$ & 1330 & 1532 & 1900 & 1766 & 1973 & 2020 & 1956 & $\begin{array}{l}\text { (15) } \\
1909\end{array}$ & $\begin{array}{l}\text { (16) } \\
1507\end{array}$ & 1662 & 1704 & 1726 & 1712 & 1694 & $\begin{array}{l}1594 \\
1594\end{array}$ & $\begin{array}{l}1693 \\
1693\end{array}$ & $\begin{array}{l}\text { (17) } \\
1308\end{array}$ & (19) \\
\hline (ppm) & (3) & (4) & (3) & (5) & (7) & (5) & (7) & (13) & (14) & (9) & (14) & (24) & (27) & $\begin{array}{l}1694 \\
(23)\end{array}$ & (III) & (13) & 1308 & 1643 \\
\hline $\mathrm{Ni}$ & 15 & 12 & 21 & 1. & 21 & I & 20 & 36 & 63 & 91 & 48 & 30 & 38 & 36 & 100 & 55 & 126 & (17) \\
\hline (ppm) & (3) & (4) & (3) & (5) & (7) & (5) & (7) & (13) & (14) & (9) & (14) & $(24)$ & (27) & (23) & (11) & (13) & (16) & (17) \\
\hline $\mathrm{Fe}_{2} \mathrm{O}_{3}$ & 0.72 & 0.74 & 1.00 & 0.82 & 0.70 & 0.73 & 0.75 & 0.90 & 1.24 & 0.98 & 0.91 & 0.65 & 0.54 & 0.49 & 0.91 & 0.79 & 0.90 & 1.02 \\
\hline (wi\%) & (3) & (4) & (3) & (5) & (7) & (5) & (7) & (13) & (14) & (9) & (14) & (24) & (27) & (23) & (11) & (13) & (16) & (17) \\
\hline $\mathrm{Co}$ & 4 & 5 & 8 & 9 & 7 & 7 & 7 & 8 & 12 & 14 & 12 & 11 & 10 & 9 & 15 & 10 & 15 & 13 \\
\hline (ppm) & (3) & (4) & (3) & (5) & (7) & (5) & (6) & (13) & (14) & (9) & (14) & (24) & (27) & (23) & (ii) & (13) & (16) & (17) \\
\hline $\mathrm{MnO}_{2}$ & 0.12 & 0.11 & 0.24 & 0.16 & 0.14 & 0.10 & 0.19 & 0.13 & 0.17 & 0.17 & 0.20 & 0.15 & 0.11 & 0.09 & 0.20 & 0.17 & 0.22 & 0.22 \\
\hline$(w t \%)$ & (3) & (4) & (3) & (5) & (7) & (5) & (7) & (13) & (14) & (9) & (14) & (24) & (27) & (23) & (11) & (13) & (16) & (17) \\
\hline $\mathrm{v}$ & 47 & 42 & 60 & 54 & 57 & 60 & 56 & 54 & 58 & 60 & 54 & 51 & 48 & 46 & 55 & 45 & 56 & 56 \\
\hline (ppm) & (3) & (4) & (3) & (5) & (7) & (5) & (7) & (13) & (14) & (9) & (14) & (24) & (27) & (23) & (ii) & (13) & & (17) \\
\hline $\mathrm{Ba}$ & 0.17 & 0.12 & 0.14 & 0.15 & 0.15 & 0.13 & 0.14 & 0.20 & 0.29 & 0.24 & 0.16 & 0.11 & 0.10 & 0.11 & 0.19 & 0.16 & 0.20 & 0.19 \\
\hline$(w t \%)$ & (3) & (4) & (3) & (5) & (7) & (5) & (7) & (13) & (14) & (9) & (14) & (24) & (26) & (23) & (1I) & (13) & (16) & (17) \\
\hline $\mathrm{TiO}_{2}$ & 0.06 & 0.08 & 0.07 & 0.07 & 0.06 & 0.06 & 0.07 & 0.07 & 0.08 & 0.07 & 0.08 & 0.07 & 0.06 & 0.06 & 0.08 & 0.07 & 0.08 & 0.09 \\
\hline$(w 1 \%)$ & (3) & (4) & (3) & (5) & (7) & (5) & (7) & (13) & (14) & (9) & (14) & (24) & (27) & (23) & (11) & (13) & & (17) \\
\hline $\mathrm{Zn}$ & - & 80 & - & - & 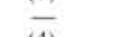 & - & - & 90 & 190 & 187 & 85 & 125 & 105 & 100 & 137 & 126 & 163 & 118 \\
\hline (ppm) & (1) & & & (2) & (4) & (3) & (4) & (2) & (4) & (2) & (6) & (5) & (7) & (7) & & & & \\
\hline $\mathrm{Cu}$ & 37 & 63 & 70 & 5 & 6 & 85 & & 59 & 94 & 88 & 62 & 65 & 57 & 63 & 71 & 59 & 102 & 68 \\
\hline (ppm) & (3) & & & (3) & (3) & (2) & (4) & (II) & (14) & (9) & (14) & (20) & (23) & & & (9) & & \\
\hline $\mathrm{Fe} / \mathrm{Mn}$ & 6.18 & 6.78 & 5.69 & 5.69 & 5.93 & 7.49 & 5.12 & 6.36 & 7.14 & 6.09 & 5.24 & 5.38 & 5.14 & 5.71 & 5.13 & 5.41 & 6.71 & 5.45 \\
\hline $\mathrm{MgO}$ & & (4) 0.37 & $\begin{array}{l}\text { (3) } \\
0.51\end{array}$ & $\begin{array}{l}\text { (5) } \\
0.48\end{array}$ & (7) 0.50 & $\begin{array}{l}\text { (5) } \\
0.40\end{array}$ & $\begin{array}{l}(8) \\
0.51\end{array}$ & 0.47 & $\begin{array}{l}\text { (16) } \\
0.54\end{array}$ & $\begin{array}{l}(9) \\
0.50\end{array}$ & $\begin{array}{l}\text { (14) } \\
0.43\end{array}$ & $\begin{array}{l}(29) \\
0.40\end{array}$ & (31) & $\begin{array}{l}(30) \\
0.36\end{array}$ & (18) & 0.50 & 0.54 & ${ }^{(20)}{ }^{-50}$ \\
\hline (wt\%) & (3) & (4) & (3) & (5) & (7) & (5) & (8) & (15) & (16) & (9) & (14) & (28) & (29) & (30) & (17) & (16) & (17) & (19) \\
\hline $\mathrm{SiO}_{2}$ biog. & 25.53 & 12.63 & $\begin{array}{l}7.59 \\
(3)\end{array}$ & $\begin{array}{l}15.46 \\
(5)\end{array}$ & 12.17 & $\begin{array}{l}10.44 \\
(5)\end{array}$ & $\begin{array}{l}12.95 \\
(8)\end{array}$ & $\begin{array}{l}24.62 \\
(15)\end{array}$ & $\begin{array}{l}29.42 \\
(16)\end{array}$ & $\begin{array}{l}23.82 \\
(9)\end{array}$ & $\begin{array}{l}15.33 \\
(14)\end{array}$ & $\begin{array}{l}17.58 \\
(28)\end{array}$ & $\begin{array}{l}15.66 \\
(29)\end{array}$ & $\begin{array}{l}17.24 \\
(30)\end{array}$ & $\begin{array}{l}17.14 \\
(18)\end{array}$ & $\begin{array}{l}15.27 \\
(16)\end{array}$ & $\begin{array}{l}23.89 \\
(17)\end{array}$ & $\begin{array}{l}13.18 \\
\text { (19) }\end{array}$ \\
\hline $\mathrm{CaCO}_{3}$ & 57.12 & 78.52 & 80.19 & 73.96 & 78.12 & 81.00 & 77.05 & 61.61 & 54.64 & 62.11 & 74.24 & 71.49 & 73.62 & 73.33 & 66.10 & 69.81 & 56.42 & 70.00 \\
\hline & (3) & (4) & & & (7) & (5) & & (15) & (16) & & & (28) & (29) & (30) & & & & (19) \\
\hline OCFM & 17.13 & 8.78 & 12.93 & 10.44 & 9.74 & 8.62 & 10.01 & $\begin{array}{l}13.23 \\
15 .\end{array}$ & 15.23 & 13.63 & 10.31 & 10.66 & 10.60 & 9.02 & 17.16 & $\begin{array}{l}14.68 \\
016\end{array}$ & 19.85 & 17.57 \\
\hline & (3) & (4) & & & & & & (15) & & & & & & & & & & \\
\hline $\mathrm{Fe}+\mathrm{Mn}$ & 13.66 & 10.58 & 16.16 & 14.32 & 12.65 & 12.00 & 11.96 & 14.98 & 17.76 & 16.29 & 12.60 & 10.64 & 11.16 & 9.75 & 12.63 & 14.00 & 13.55 & 11.08 \\
\hline$M \mathrm{P}$ & & & & (5) & (7) & (5) & & (17) & (16) & & & (29) & (31) & & (18) & & & (20) \\
\hline $\begin{array}{c}\mathrm{MAR} \\
\mathrm{CaCCO}_{3}\end{array}$ & ${ }_{(3)}^{1.7023}$ & (4) 2.4741 & ${ }_{(3)}^{1.3363}$ & $\begin{array}{l}1.2303 \\
(5)\end{array}$ & ${ }_{(7)}^{1.8008}$ & ${ }_{(5)}^{2.0038}$ & ${ }_{(8)}^{1.9226}$ & ${ }_{(15)}^{1.7561}$ & ${ }_{(16)}^{1.2920}$ & $\begin{array}{l}1.2088 \\
(9)\end{array}$ & ${ }_{(14)^{1.5351}}$ & ${ }_{(28)}^{2.4001}$ & ${ }^{2.25)}$ & $\begin{array}{l}3.1277 \\
(30)\end{array}$ & ${ }_{(18)^{1.2794}}$ & $\begin{array}{c}0.9711 \\
(16)\end{array}$ & $\begin{array}{l}0.7114 \\
\text { (17) }\end{array}$ & $\begin{array}{l}1.0107 \\
\text { (19) }\end{array}$ \\
\hline $\begin{array}{c}(\mathrm{g} / \mathrm{cm}-\mathrm{k} . \mathrm{y} .) \\
\mathrm{MAR} \\
\mathrm{SiO}_{2} \text { biog. }\end{array}$ & $\underset{(3)}{0.7608}$ & $\begin{array}{l}0.3980 \\
(4)\end{array}$ & $\begin{array}{l}0.1265 \\
\text { (3) }\end{array}$ & $\begin{array}{l}0.2572 \\
(5)\end{array}$ & $\begin{array}{l}0.2805 \\
(7)\end{array}$ & $\begin{array}{l}0.2583 \\
\text { (5) }\end{array}$ & $\begin{array}{l}0.3231 \\
(8)\end{array}$ & $\begin{array}{l}0.7018 \\
\text { (15) }\end{array}$ & $\begin{array}{l}0.6956 \\
\text { (16) }\end{array}$ & $\begin{array}{l}0.4636 \\
(9)\end{array}$ & $\begin{array}{l}0.3170 \\
(14)\end{array}$ & $\begin{array}{l}0.5902 \\
(28)\end{array}$ & $\begin{array}{l}0.4796 \\
\text { (29) }\end{array}$ & $\begin{array}{l}0.7353 \\
(30)\end{array}$ & $\begin{array}{l}0.3318 \\
(18)\end{array}$ & $\begin{array}{l}0.2194 \\
(16)\end{array}$ & $\begin{array}{l}0.3012 \\
\text { (17) }\end{array}$ & $\begin{array}{l}0.1903 \\
(19)\end{array}$ \\
\hline $\begin{array}{c}\left(\mathrm{g} / \mathrm{cm}^{2} / \mathrm{k} . \mathrm{y} .\right) \\
\text { MAR }\end{array}$ & 05105 & & 0.2155 & 0.1737 & & & & 0.3771 & 0.3601 & 0.2653 & 0.2132 & 0.3579 & 0.3247 & 0.3847 & 0.3321 & 0.2042 & 0.2503 & 0.2537 \\
\hline OCFM & (3) & (4) & (3) & (5) & (7) & (5) & (8) & (15) & (16) & (9) & (14) & (28) & (29) & (30) & (18) & (16) & (17) & (19) \\
\hline $\begin{array}{l}\text { MAR } \\
\mathrm{Ba}\end{array}$ & $\begin{array}{l}0.0051 \\
(3)\end{array}$ & $\begin{array}{l}0.0034 \\
(4)\end{array}$ & $\begin{array}{l}0.0022 \\
(3)\end{array}$ & $\begin{array}{l}0.0028 \\
(5)\end{array}$ & $\begin{array}{l}0.0035 \\
(7)\end{array}$ & $\begin{array}{l}0.0033 \\
(5)\end{array}$ & $\begin{array}{l}0.0034 \\
(7)\end{array}$ & $\begin{array}{l}0.0036 \\
(13)\end{array}$ & $\begin{array}{l}0.0033 \\
(14)\end{array}$ & $\begin{array}{l}0.0036 \\
(9)\end{array}$ & $\begin{array}{l}0.0027 \\
(13)\end{array}$ & $\begin{array}{l}0.0030 \\
(22)\end{array}$ & $\begin{array}{l}0.0029 \\
(25)\end{array}$ & $\begin{array}{l}0.0040 \\
(23)\end{array}$ & $\begin{array}{l}0.0034 \\
\text { (11) }\end{array}$ & $\begin{array}{l}0.0024 \\
(12)^{0}\end{array}$ & $\begin{array}{l}0.0032 \\
\text { (15) }\end{array}$ & $\begin{array}{l}0.0022 \\
(16)\end{array}$ \\
\hline $\begin{array}{c}\left(\mathrm{g} / \mathrm{cm}^{2} / \mathrm{y} .\right) \\
\mathrm{MAR} \\
\mathrm{Fe} \\
\left(\mathrm{g} / \mathrm{cm}^{2} / \mathrm{k} . \mathrm{y} .\right)\end{array}$ & $\begin{array}{l}0.0214 \\
\text { (3) }\end{array}$ & $\begin{array}{l}0.0258 \\
(4)\end{array}$ & $\begin{array}{l}0.0146 \\
(3)\end{array}$ & $\begin{array}{l}0.0124 \\
(5)\end{array}$ & $\begin{array}{l}0.0134 \\
(7)\end{array}$ & $\begin{array}{l}0.0160 \\
(5)\end{array}$ & $\begin{array}{l}0.0148 \\
(7)\end{array}$ & $\begin{array}{l}0.0186 \\
(13)\end{array}$ & $\begin{array}{l}0.0193 \\
(14)\end{array}$ & $\begin{array}{l}0.0164 \\
(9)\end{array}$ & $\begin{array}{l}0.0116 \\
(14)\end{array}$ & $\begin{array}{l}0.0146 \\
(24)\end{array}$ & $\begin{array}{l}0.0138 \\
(27)\end{array}$ & (II) 0.0172 & $\begin{array}{l}0.0162 \\
\text { (II) }\end{array}$ & $\begin{array}{l}0.0103 \\
(13)\end{array}$ & $\begin{array}{l}0.0122 \\
(15)\end{array}$ & $\begin{array}{l}0.0097 \\
\text { (16) }\end{array}$ \\
\hline
\end{tabular}


Table 5. Average concentrations of the elements (on OCFM) in F3 sediments and other ancient and recent metalliferous sediments of the Pacific Ocean.

\begin{tabular}{|c|c|c|c|c|c|c|}
\hline Element & $\begin{array}{c}(1) \\
N=22\end{array}$ & $\begin{array}{c}\text { DSDP Sites } \\
82,83 \\
(2) \\
N=16\end{array}$ & $\begin{array}{c}\text { DSDP Sites } \\
573,574 \\
(3) \\
N=44\end{array}$ & $\begin{array}{c}\text { Pacific } \\
\text { J3-Q } \\
(4) \\
N=920\end{array}$ & $\begin{array}{c}\mathrm{EPR} 5^{\circ}-40^{\circ} \mathrm{S} \\
\text { recent } \\
\quad(5) \\
N=235\end{array}$ & $\begin{array}{c}\text { EPR } 11^{\circ}-14^{\circ} \mathrm{N} \\
\text { recent } \\
(6) \\
N=91\end{array}$ \\
\hline $\mathrm{Fe}(\%)$ & 11.87 & 12.8 & 14.4 & 19.2 & 19.4 & 12.79 \\
\hline $\mathrm{Mn}(\%)$ & 2.07 & 2.1 & 3.17 & 4.0 & 5.8 & 2.65 \\
\hline $\mathrm{Al}(\%)$ & 5.17 & 4.6 & 3.68 & 4.0 & 3.8 & 5.24 \\
\hline $\mathrm{Ti}(\%)$ & 0.36 & & 0.32 & 0.28 & 0.31 & 0.29 \\
\hline $\mathrm{Cu}(\mathrm{ppm})$ & 439 & 495 & 963 & 820 & 1200 & 335 \\
\hline $\mathrm{Zn}(\mathrm{ppm})$ & 438 & 580 & 541 & 560 & 470 & 371 \\
\hline $\mathrm{Ni}(\mathrm{ppm})$ & $\begin{array}{l}430 \\
332\end{array}$ & 340 & 505 & 520 & 950 & 245 \\
\hline Co (ppm) & 76 & 34 & 87 & 80 & 250 & 110 \\
\hline $\mathrm{V}(\mathrm{ppm})$ & 361 & & 228 & 410 & 490 & 288 \\
\hline $\mathrm{Ba}(\mathrm{ppm})$ & 10,830 & 7,800 & 14,800 & 5,300 & 12,200 & 4,000 \\
\hline
\end{tabular}

Note: Sources of data are (1) our data; (2) Donnelly, 1980a, and our data; (3) Jarvis, 1985; (4) and (5) Lisitzin et al., 1992; (6) Cherkashev, 1992. $N=$ number of analyses.

Table 6. Mean ratios of element/aluminum in clayey matter and concentrations of elements in biogenic carbonate and silica matter of sediments.

\begin{tabular}{cccc}
\hline & $\begin{array}{c}\text { Element/aluminum } \\
\text { Element }\end{array}$ & $\begin{array}{c}\text { Carbonate matter } \\
(1)\end{array}$ & $\begin{array}{c}\text { Silica matter } \\
(2)\end{array}$ \\
\hline $\mathrm{Fe}$ & 0.54 & 510 & $(3)$ \\
$\mathrm{Mn}$ & 0.051 & 160 & 350 \\
$\mathrm{Cu}$ & 0.0017 & 21 & 4 \\
$\mathrm{Zn}$ & 0.0011 & 10 & 7 \\
$\mathrm{Ni}$ & 0.0013 & 20 & 2 \\
$\mathrm{Co}$ & 0.00031 & 5 & 0.5 \\
$\mathrm{~V}$ & 0.0017 & 10 & 1 \\
$\mathrm{Ba}$ & 0.032 & 20 & 20 \\
\hline
\end{tabular}

Notes: Sources of data are (1) Turekian and Wedepohl, 1961; Strakhov, 1976; Jarvis, 1985; (2) Bogdanov et al., 1983; our new data; (3) Martin and Knauer, 1973; Bogdanov et al., 1983; our new data. All concentrations in parts per million.

nonmetalliferous sediments are enriched in Ba (Boström et al., 1973; Gurvich et al., 1978, 1979). In metalliferous sediments of the East Pacific Rise, beneath the low productivity zone, nonhydrothermal barite and hydrothermal particles are the main carriers of $\mathrm{Ba}$. Near the EPR axis, about $50 \%$ of the Ba content combines with the hydrothermal matter and about $40 \%$ combines with nonhydrothermal barite. On the flanks of the EPR, only about $15 \%$ of the Ba content combines with hydrothermal matter and about $80 \%$ with nonhydrothermal barite (Gurvich et al., 1979). In the high productivity zone, near the equator, $\mathrm{Ba}$ and nonhydrothermal barite accumulation rates are 5 to 10 times higher than rates in the low productivity zone (Boström et al., 1973; Gurvich et al., 1978), and nonhydrothermal barite may be the greater component in metalliferous sediments. Most of the excess $\mathrm{Ba}$ has accumulated as barite in the F3 sediments independently of the hydrothermal matter. This results in a decrease of the ratio $\mathrm{Ba} /\left(\mathrm{Fe}_{e}+\right.$ $\mathrm{Mn}_{e}$ ), with increasing hydrothermal MAR, because the hydrothermal particles have diluted the nonhydrothermal barite.

Factor 2 explains $36.6 \%$ of the variance (Fig. 2). $\mathrm{Fe}^{\prime}, \mathrm{Zn}^{\prime}$, and $\mathrm{V}^{\prime}$ have significant positive values; $\mathrm{Mn}^{\prime}$ and $\mathrm{Co}^{\prime}$ have significant negative values. The values of other parameters are insignificant. This may reflect the distribution of the elements in hydrothermal matter. Fe and $\mathrm{Mn}$ are the main components of hydrothermal matter and dilute each other. Their ratio in hydrothermal matter does not depend (or depends only slightly) on the hydrothermal MAR. Zn and V tend to combine with iron minerals. Co combines with manganese minerals. $\mathrm{Cu}$ and $\mathrm{Ni}$ may combine with either iron or manganese minerals. The insignificant value of $\mathrm{Ba}^{\prime}$ suggests that the hydrothermal matter has a relatively low content of $\mathrm{Ba}$ and that nonhydrothermal barite is the main carrier of this element. If most excess $\mathrm{Ba}$ were to combine with the hydrothermal matter, we would expect a significant negative value of $\mathrm{Ba}^{\prime}$, because in $\mathrm{Fe}-\mathrm{Mn}$ oxides, Ba preferentially combines with manganese minerals (Fisher and Puchelt, 1972; Bonatti et al., 1972; Gurvich et al., 1979).

\section{HISTORY OF HYDROTHERMAL ACTIVITY}

To study the history of the accumulation of hydrothermal material, we used the hydrothermal $\mathrm{Fe}$ accumulation rates in metalliferous and in nonmetalliferous sediments. The calculation procedure was the same as for excess Fe, but we used both our chemical data and the bulk-sediment MAR data for Leg 138 (Mayer, Pisias, Janecek, et al., 1993). For Sites 848 and 850 , we also used chemical data from Mayer, Pisias, Janecek, et al. (1972). In addition, we used data for Site 83 (DSDP Leg 9) (Hays et al., 1972; Donnelly, 1980b; our data). We used only reliable accumulation rate data. Data were rejected if they showed "sharp jumps" of the hydrothermal Fe accumulation rates or of $\mathrm{CaCO}_{3}$, opal, and $\mathrm{Al}$ accumulation rates. We rejected data having "strange" contents of $\mathrm{Fe}(\mathrm{OCFM})$ and $\mathrm{Al}(\mathrm{OCFM})$, situated far from the mixture line of clay and hydrothermal material. The reliable hydrothermal $\mathrm{Fe}$ accumulation rates $\left(\mathrm{Fe}_{h} \mathrm{MAR}\right)$ were averaged for every $0.5 \mathrm{Ma}$ and plotted along backtracked paths. The data for the western transect are shown in isopleth form and demonstrate temporal and spatial changes of $\mathrm{Fe}_{h}$ MAR on the western flank of the EPR (Fig. 3). Data for ODP Sites 845 and 846 and DSDP Site 83 are shown as curves only.

Figure 3 illustrates that the basal sediments usually have the highest $\mathrm{Fe}_{h}$ MAR. These values are comparable with those observed near the crest of the EPR, in the main fields of recent metalliferous sediments and in basal metalliferous sediments recovered during DSDP Legs 9, 16, and 85 (Boström, 1973; Bogdanov et al., 1979; Leinen and Stakes, 1979; Jarvis, 1985; Lisitzin et al., 1990). The values are less than those reported for basal metalliferous sediments recovered during DSDP Leg 92 (Lyle et al., 1986; Rea and Leinen, 1986). Sediments from Sites 848 and 851 have the highest values of $\mathrm{Fe}_{h}$ MAR above the basal layer; however, their magnitudes are less than those in basal layers at other sites.

Assuming no redeposition of sediments on the seafloor, $\mathrm{Fe}_{h}$ MAR depends on three main factors: (1) intensity of hydrothermal influx to seawater, (2) distance from the source, and (3) bottom currents. The general relationship of $\mathrm{Fe}_{h}$ MAR to spreading rate and distance from the EPR crest for the main field of recent metalliferous sediments in the southeastern Pacific region is shown in Figure 4.

\section{7 to $11 \mathrm{Ma}$}

Few $\mathrm{Fe}_{h}$ MAR data are available for this age range; hence, its history can be described only in general terms. $\mathrm{Fe}_{h} \mathrm{MAR}$ in the basal layers of sediments at Sites 845 and 846 was equal to or more than 50 $\mathrm{mg} / \mathrm{cm}^{2} / \mathrm{k}$.y. This indicates intense hydrothermal activity from 17 to $16 \mathrm{Ma}$. Maximal $\mathrm{Fe}_{h}$ MAR occurred during 18 to $17 \mathrm{Ma}$, when this area was at the EPR at $19^{\circ} \mathrm{S}$ (Lyle et al., 1986; Rea and Leinen, 1986; 


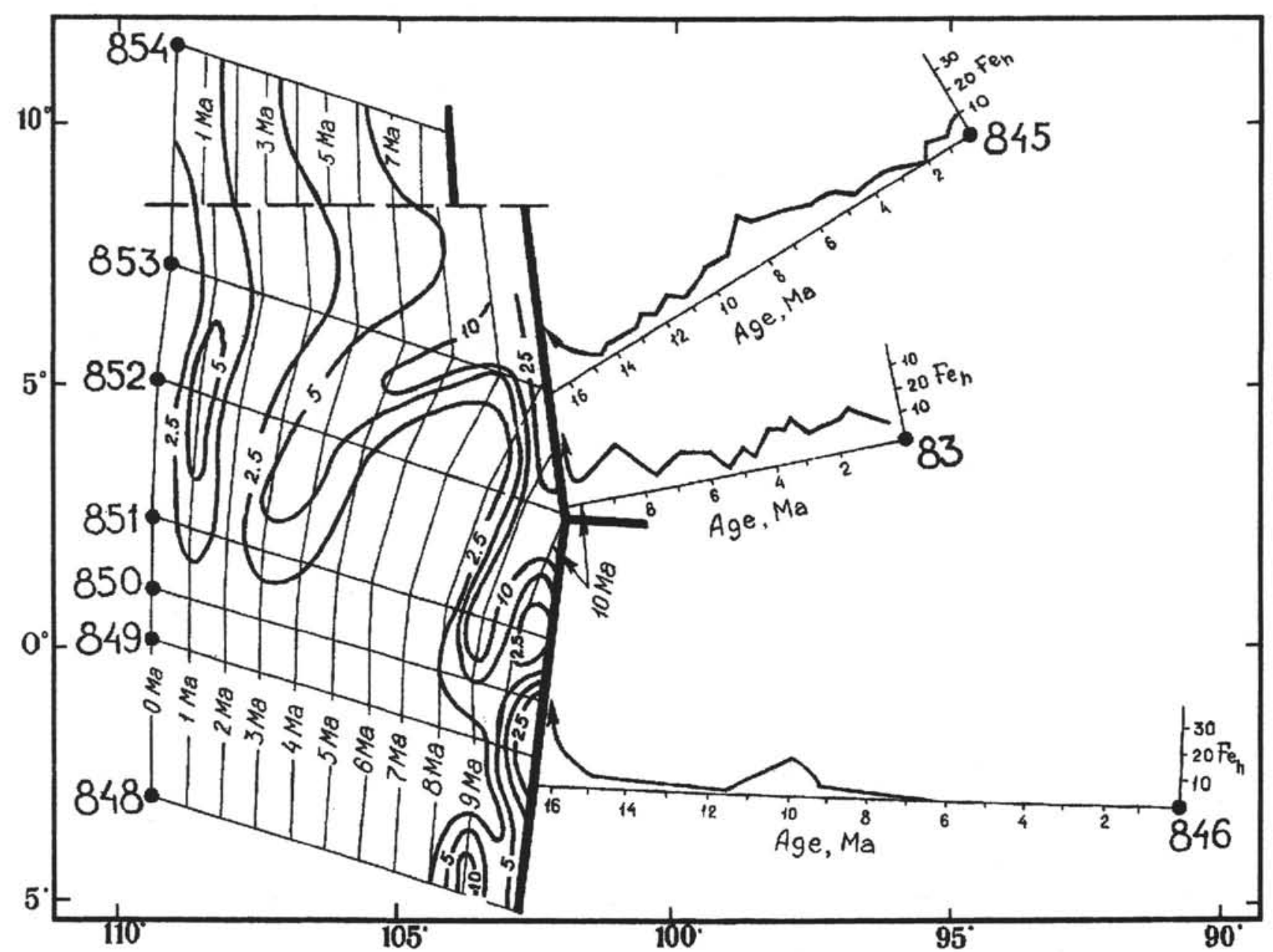

Figure 3. Distribution of hydrothermal Fe accumulation rates $\left(\mathrm{mg} / \mathrm{cm}^{2} / \mathrm{k} . \mathrm{y}\right.$.) in sediments from Sites $845,836,848$ through 854 , and $83 \mathrm{vs.} \mathrm{age} \mathrm{on} \mathrm{backtracked} \mathrm{paths.}$

Lisitzin et al., 1990), which correlates with the proposed jump of the spreading center from Gallego Rise to the EPR (Mammerickx et al., 1980). Later, about 15 to $14 \mathrm{Ma}$ in Site 845 sediments and about 15 to $12.5 \mathrm{Ma}$ in Site 846 sediments, the $\mathrm{Fe}_{h}$ MAR decreased because of an increase in the distance from the spreading center to the less intense hydrothermal activity (Leinen and Stakes, 1979; Lisitzin et al., 1990). This was the time of a minimal spreading rate in the northern Pacific (Rich et al., 1986). At about 13 to $12 \mathrm{Ma}$, a slight increase of $\mathrm{Fe}_{h}$ MAR occurred in Site 845 sediments. This correlates with a general increase in hydrothermal activity in the area (Lisitzin et al., 1990) and may be explained by plate reorganization in the northeastern tropical Pacific Ocean (Mammerickx and Klitgord, 1982).

\section{1 to $10 \mathrm{Ma}$}

The $\mathrm{Fe}_{h}$ MAR and the history of hydrothermal activity are better known from this time. Basal sediments accumulated at Sites 848 through 851 and 83 at this time. However, no high $\mathrm{Fe}_{h}$ MAR is seen in the basal sediments at Sites 848 and 851 , indicating that no nearby hydrothermal activity took place. High $\mathrm{Fe}_{h}$ MAR values (up to 30-60 $\mathrm{mg} / \mathrm{cm}_{2} / \mathrm{k}$.y. at $10.5-10 \mathrm{Ma}$ ) in the basal sediments at Sites 849 and 850 indicate that these sediments accumulated near hydrothermal fields. During this time, Site 846 was about $500 \mathrm{~km}$ away from the ridge crest. The site has a high $\mathrm{Fe}_{h}$ MAR (about $15 \mathrm{mg} / \mathrm{cm}_{2} / \mathrm{k}$.y.) for such a distance (see Figs. 3 and 4). This may indicate that the hydrothermal fields were active and that much of the suspended hydrothermal matter was transported eastward by a strong bottom current. The $\mathrm{Fe}_{h}$ MAR data for Sites 846,849 , and 850 show that the hydrothermal fields were active for about $2 \mathrm{Ma}$ : from 11.5 to 11 and 9.5 to $9 \mathrm{Ma}$, with the highest hydrothermal activity at about $10 \mathrm{Ma}$. High $\mathrm{Fe}_{h}$ MARs (about $50 \mathrm{mg} / \mathrm{cm}^{2} / \mathrm{k}$.y.) in basal sediments at Site 83 (Fig. 3) show that hydrothermal activity existed near this site at about 10.5 $\mathrm{Ma}$; however, this had decreased by $10 \mathrm{Ma}$. At $10 \mathrm{Ma}$, the $\mathrm{Fe}_{h}$ MAR in sediments at Site 845 was high, although the ridge crest was about $400 \mathrm{~km}$ away (Fig. 3). This suggests that hydrothermal activity also existed to the north and that a significant part of the suspended hydrothermal particles were transported to the east from the rise crest.

\section{5 to $8 \mathrm{Ma}$}

This was the time of the highest $\mathrm{Fe}_{h}$ MAR in nonbasal sediments at Sites 848 and 851 and of increasing $\mathrm{Fe}_{h}$ MAR in nonbasal sediments at Sites 845, 849, and 83 (Fig. 3). Evidence for such an increase is absent at Site 849 . High $\mathrm{Fe}_{h}$ MARs (more than $50 \mathrm{mg} / \mathrm{cm}^{2} / \mathrm{k} . \mathrm{y}$.) occurred at $9 \mathrm{Ma}$ in basal sediments at Site 83 (Leinen and Stakes, 1979; Lisitzin et al., 1990). Its backtracked path lies between those of Sites 851 and 852. This distribution of $\mathrm{Fe}_{h}$ MAR indicates that an increase of hydrothermal activity between $4^{\circ}$ and $5^{\circ} \mathrm{S}$ and between $1^{\circ}$ and, at least, $5^{\circ} \mathrm{N}$ occurred between 9.5 and $8 \mathrm{Ma}$. Near $3^{\circ} \mathrm{N}, \mathrm{Fe}_{h}$ MARs on the eastern flank were higher than those on the western flank at equal distances from the ridge crest (Fig. 3). This again indicates the eastward transport of suspended hydrothermal matter. About $9 \mathrm{Ma}$ was the time of increased hydrothermal activity at the EPR at $19^{\circ} \mathrm{S}$. This may correlate with the plate-tectonic reorientation event between 10.5 and $8 \mathrm{Ma}$ marked the change in strike of the PacificRiviera Ridge from north-northwest to northeast (Lyle et al., 1986; 


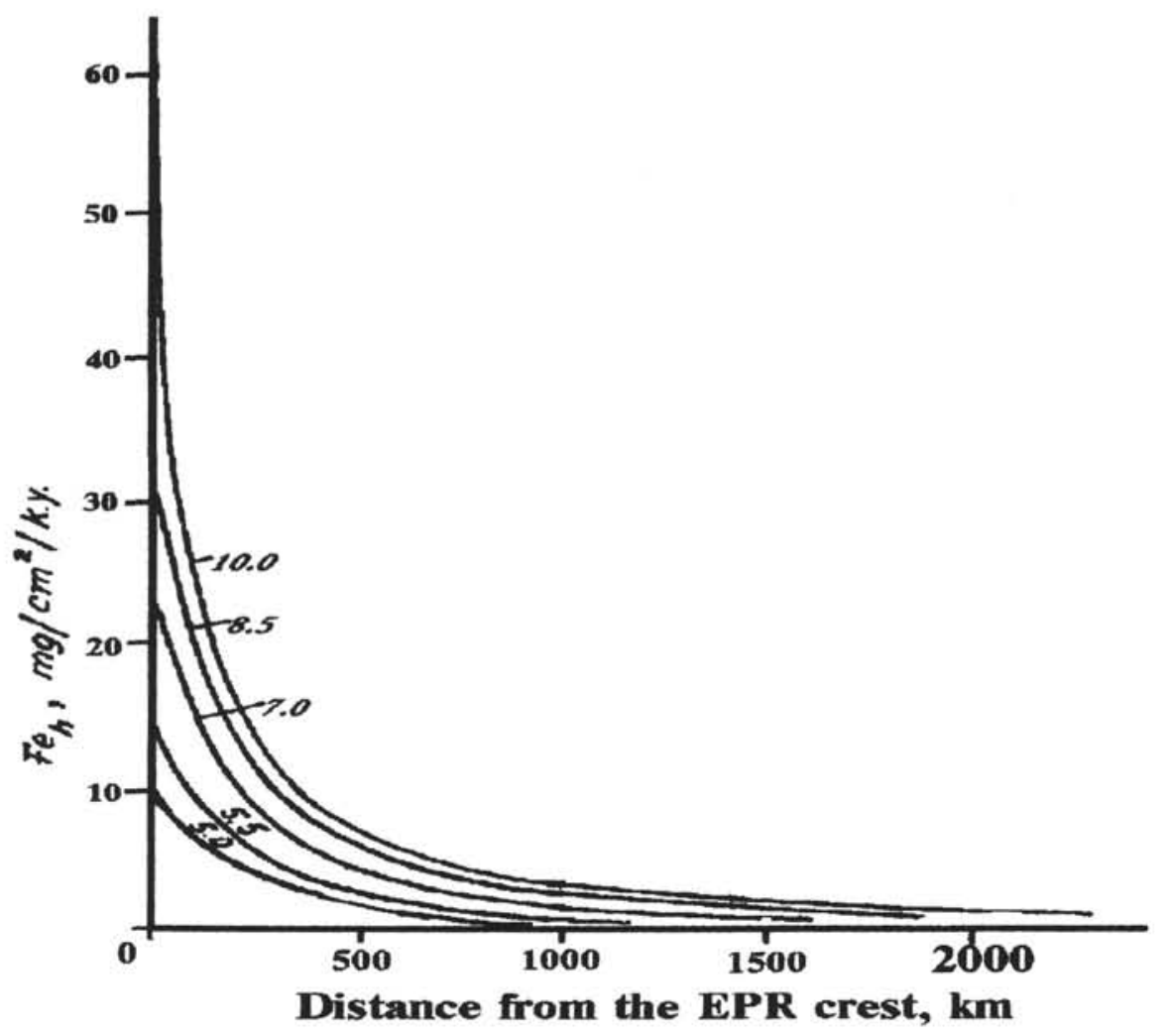

Figure 4. Hydrothermal Fe accumulation rate in surface metalliferous sediments at the East Pacific Rise between $5^{\circ}$ and $45^{\circ} \mathrm{S}$ as a function of distance from the rise crest and spreading rate. Figures near the lines indicate half-spreading rates (after E. Gurvich in Lisitzin et al., 1990,with modification).

Rea and Leinen, 1986). About 9 to $8 \mathrm{Ma}$ was the time of the highest mean spreading rate for the North Pacific for the last $15 \mathrm{Ma}$ of its history (Rich et al., 1986).

\section{8 to $5 \mathrm{Ma}$}

$\mathrm{Fe}_{h}$ MARs in sediments at Sites 846 and 848 through 852 were low, or $\mathrm{Fe}_{h}$ is absent. During this time, little hydrothermal activity was taking place in the southern half of the EPR study area. $\mathrm{Fe}_{h}$ MARs in sediments at Sites $83,845,853$, and 854 indicate activity in the northern half of the study area. The most intensive activity was located between $5^{\circ}$ and $10^{\circ} \mathrm{N}$. Eastward transport of suspended hydrothermal matter dominated.

\section{5 to $3 \mathrm{Ma}$}

$\mathrm{Fe}_{h}$ MARs in sediments at Sites 846 and 848 through 850 were low, or $\mathrm{Fe}_{h}$ is absent. About 4 to $3 \mathrm{Ma}, \mathrm{Fe}_{h} \mathrm{MAR}$ at Site 851 was 2.5 to $5 \mathrm{mg} / \mathrm{cm}^{2} / \mathrm{k}$.y. To the north, at Site 852 , an increased $\mathrm{Fe}_{h}$ MAR occurred from 5 to $3 \mathrm{Ma}$ and reached maximal values at 4.5 to $3.5 \mathrm{Ma}$. In Site 83 sediments, the $\mathrm{Fe}_{h}$ MAR also increased between 4 and 3 $\mathrm{Ma}$. The $\mathrm{Fe}_{h}$ MARs decreased gradually at Sites 853,854 , and 845 between 5 and $3 \mathrm{Ma}$. This distribution of $\mathrm{Fe}_{h}$ MAR indicates that from 5 to $3 \mathrm{Ma}$, hydrothermal activity in the southern part of the EPR section under study was weak. On the EPR between $7^{\circ}$ and $2^{\circ}-3^{\circ} \mathrm{N}$, the maximum hydrothermal activity moved along the axis from $5^{\circ}$ to $6^{\circ} \mathrm{N}$, to the area of triple junction, a distance of 300 to $350 \mathrm{~km}$. The mean rate of the movement was 15 to $18 \mathrm{~cm} / \mathrm{yr}$. For comparison, the rates of movement of hydrothermal fields along the EPR axis, between $20^{\circ} 30^{\prime}$ and $22^{\circ} \mathrm{S}$, have been estimated to be about 40 to 80 $\mathrm{cm} / \mathrm{yr}$ for the last $50,000 \mathrm{yr}$, with the mean rate being about $70 \mathrm{~cm} / \mathrm{yr}$ (Dekov, 1992).

Increasing hydrothermal activity on the EPR at $19^{\circ} \mathrm{S}$ has been postulated in the time interval from 6 to $3 \mathrm{Ma}$ as well (Lyle at al., 1986).
Comparison of $\mathrm{Fe}_{h}$ MAR on the eastern and western flanks of the EPR between 5 and $3 \mathrm{Ma}$ (Fig. 3) indicates that the eastward transport of suspended hydrothermal matter was not as great as before.

\section{3 to $2 \mathrm{Ma}$}

$\mathrm{Fe}_{h}$ MARs were low or zero in sediments of all sites in the study area, except at Site 83, where the $\mathrm{Fe}_{h}$ MAR decreased (Fig. 3). During this period, the center of hydrothermal activity was near the triple junction, or along the Galapagos spreading center.

\section{2 to $0 \mathrm{Ma}$}

$\mathrm{Fe}_{h}$ MARs at Sites 846 and 848 through 850 were low or zero. At Sites 851 through $853,700 \mathrm{~km}$ from the EPR crest, values of $\mathrm{Fe}_{h}$ MAR increased $\left(2.5-7 \mathrm{mg} / \mathrm{cm}^{2} / \mathrm{k} . \mathrm{y}\right.$.) between 1.5 and $0.5 \mathrm{Ma}$, with a maximum near $1 \mathrm{Ma}$. At Site 854 , values of $\mathrm{Fe}_{h} \operatorname{MAR}\left(2.5-4 \mathrm{mg} / \mathrm{cm}^{2} / \mathrm{k}\right.$.y.) increased from 2-1.5 Ma to at least 0.3 Ma. At Site 845, on the eastern flank of the EPR, the $\mathrm{Fe}_{h}$ MAR increased as well. These increases were apparently caused by hydrothermal activity at the EPR crest to the north of the Siqueiros Fracture Zone. The existence of this activity is confirmed by data obtained during DSDP Leg 54 (Rosendahl, Hekinian, et al., 1980). Basal sediments recovered near the Siqueiros Fracture Zone are metalliferous. These accumulated from 1.1 to 2.2 Ma. According to our estimate (made using Leg 54 data; Rosendahl, Hekinian, et al., 1980; Donnely, 1980a, 1980b; Schrader et al., 1980), the values of $\mathrm{Fe}_{h}$ MAR in these sediments are high-from tens to hundreds of milligrams per centimeters square per thousand years.

Site 854 is located at the same latitude as the high-temperature vent fields and massive sulfide deposits on the EPR crest $\left(11^{\circ}-13^{\circ} \mathrm{N}\right.$; Hekinian et al., 1985), and a field of recent metalliferous sediments lies between $9^{\circ}$ and $14^{\circ} \mathrm{N}$ (Krasnov et al., 1992). The increase in the $\mathrm{Fe}_{h}$ MAR in sediments at Sites 845 and 854 began about $2 \mathrm{Ma}$, marking the beginning of intense hydrothermal activity at the EPR 
crest north of the Siqueiros Fracture Zone, between $11^{\circ}$ and $13^{\circ} \mathrm{N}$. Increasing hydrothermal activity at the EPR at $19^{\circ} \mathrm{S}$ is supposed to have taken place in the time interval from $2 \mathrm{Ma}$ to the present as well (Lyle at al., 1986).

From $2 \mathrm{Ma}$ to the present, sediments accumulated on the eastern flank of the EPR (Sites 83 and 845) at the same distance or farther from the crest than sediments of the same age on the western flank (Sites 851 through 854 ) had higher $\mathrm{Fe}_{h}$ MAR. This again indicates the dominant eastward transport of suspended hydrothermal matter.

Data obtained for $\mathrm{Fe}_{h}$ MAR distribution on the flanks of the EPR indicate that the dominant eastward transport in the studied area (and mainly eastward direction of bottom currents) occurred from at least 10-11 Ma to the present. This result is in good accordance with present-day observations. According to hydrographic data and direct current measurements, today's abyssal water flows in an eastward direction across the EPR in this area (Lonsdale, 1976).

\section{CONCLUSIONS}

1. The sediments studied form three sedimentary sequences: siliceous-clayey (F1), siliceous-carbonate (F2), and metalliferous (F3). These sequences differ from each other by their bulk chemical composition and by their chemical composition of opal-carbonatefree matter (OCFM). OCFM of F1 and F2 is mainly terrigenous, but the relative role of eolian material is greater in F2 sediments. The OCFM of F3 sediments contains much hydrothermal material.

2. Metalliferous sediments of the F3 sequence have lower average contents of $\mathrm{Fe}$ and $\mathrm{Mn}$ and combined microelements, and higher average contents of $\mathrm{Ti}$ and $\mathrm{Al}$ than other recent and ancient metalliferous sediments of the Pacific Ocean.

$\mathrm{Cu}, \mathrm{Zn}, \mathrm{Ni}, \mathrm{Co}$, and $\mathrm{V}$ entered hydrothermal ore matter of these sediments by sorption by hydrothermal particles from seawater. Iron minerals are the main carriers of $\mathrm{Zn}$ and $\mathrm{V}$ combined with the hydrothermal matter. Manganese minerals are the main carriers of $\mathrm{Co}$ combined with the hydrothermal matter. Both iron and manganese minerals are the carriers of $\mathrm{Cu}$ and $\mathrm{Ni}$ combined with the hydrothermal matter. Barite is the main carrier of $\mathrm{Ba}$.

3. Periods of increased hydrothermal activity and the positions of centers of hydrothermal activity were as follows: 17-16 Ma, north and south of the present position of the triple junction; $10.5-9.5 \mathrm{Ma}$, between $1^{\circ}$ and $3^{\circ} \mathrm{S} ; 9.5-8 \mathrm{Ma}$, between $4^{\circ}$ and at least $5^{\circ} \mathrm{S}$, between $1^{\circ} \mathrm{S}$ and at least $5^{\circ} \mathrm{N} ; 8-5 \mathrm{Ma}$, between $5^{\circ}$ and $10^{\circ} \mathrm{N} ; 5-3 \mathrm{Ma}$, movement of the maximal hydrothermal activity along the EPR axis from $5^{\circ}$ to $6^{\circ} \mathrm{N}$ to the area of the triple junction-the mean rate of this movement was 15 to $18 \mathrm{~cm} / \mathrm{yr} ; 1.5-0.5 \mathrm{Ma}$, between Siqueiros Fracture Zone and the triple junction; $2-0 \mathrm{Ma}$, to the north from Siqueiros Fracture Zone up to $14^{\circ} \mathrm{N}$.

4. Predominantly eastward transport of suspended hydrothermal particles by bottom currents in the study area has occurred from at least $10-11 \mathrm{Ma}$ to the present.

\section{ACKNOWLEDGMENTS}

We are grateful to A.P. Lisitzin for supporting this study. Special thanks are extended to G.V. Ivanov, L.V. Demina, and N.P. Tolmacheva for providing results for minor elements, $\mathrm{CaCO}_{3}, \mathrm{SiO}_{2}, \mathrm{C}_{\text {org }}$, and $\mathrm{P}_{2} \mathrm{O}_{5}$. Work was supported by Russian Foundation of Fundamental Research Grant No. 93-05-9274.

\section{REFERENCES}

Bogdanov, Y.A., Gurvich, E.G., and Lisitzin, A.P., 1983. Mechanism of oceanic sedimentation and of differentiation of chemical elements in the

\footnotetext{
Abbreviations for names of organizations and publications in ODP reference lists follow the style given in Chemical Abstracts Service Source Index (published by American Chemical Society).
}

ocean. In Monin, A.S., and Lisitzin, A.P. (Eds.), Biogeochemistry of the Ocean: Moscow (Nauka), 165-200. (in Russian)

Bogdanov, Y.A., Lisitzin, A.P., Migdisov, A.A., Smirnov, V.I., and Starostin, V.I., 1979. On the genesis of metalliferous sediments. In V.I. Smirnov (Ed.), Metalliferous Sediments of the Southeastern Part of the Pacific Ocean: Moscow (Nauka), 249-275. (in Russian)

Bonatti, E., Fisher, D.E., Joensuu, O., Rydell, H., and Beyth, M., 1972. Iron-manganese-barium deposit from the northern Afar Rift (Ethiopia). Econ. Geol., 67:717-730.

Boström, K., 1973. The origin and fate of ferromanganoan active ridge sediments. Stockholm Contrib. Geol., 27:149-243.

Boström, K., Joensuu, O., Moore, C., Boström, B., Dalziel, M., and Horowitz, A., 1973. Geochemistry of barium in pelagic sediments. Lithos, 6:159-174.

Cherkashev, G.A., 1992. Geochemistry of metalliferous sediments from oreforming areas of the ocean. In Gramberg, I.S., and Ainemer, A.I. (Eds.), Hydrothermal Sulfide Ores and Metalliferous Sediments of the Ocean: St. Petersburg (Nedra), 138-152. (in Russian)

Dekov, V.M., 1992. Sedimentation in the areas of hydrothermal activity in the ocean (EPR, $21^{\circ} \mathrm{S}$.) [Ph.D. thesis]. P.P. Shirshov Inst. of Oceanology, Moscow. (in Russian)

Donnelly, T.W., 1980a. Chemical composition of deep sea sediments-Sites 9 through 425, Legs 2 through 54, Deep Sea Drilling Project. In Rosendahl, B.R., Hekinian, R., et al., Init. Repts. DSDP, 54: Washington (U.S. Govt. Printing Office), 899-949.

1980b. Secondarily modified sediments of the eastern Pacific: major element chemistry of Sites 420, 424, and 425, Deep Sea Drilling Project Leg 54. In Rosendahl, B.R., Hekinian, R., et al., Init. Repts. DSDP, 54: Washington (U.S. Govt. Printing Office), 329-338.

Fisher, K., and Puchelt, H., 1972. Barium. In Wedepohl, K.H. (Ed.), Handbook of Geochemistry, (Vol. VII, Pt. 3): Berlin (Springer).

Gurvich, Y.G., Bogdanov, Y.A., Kurinov, A.D., and Katargin, N.V., 1977. Scandium in bottom sediments of the southeastern Pacific. Geokhimia 10:1582-1597. (in Russian)

Gurvich, Y.G., Bogdanov, Y.A., and Lisitzin, A.P., 1979. Barium behavior in the recent sedimentation of metalliferous sediments in the Pacific ocean. Geokhimia, 3:108-125. (in Russian)

Gurvich, Y.G., Lisitzin, A.P., and Kurinov, A.D. 1980a. Hafnium. In Ronov, A.B. (Ed.), Geochemistry of Hydrolyzate Elements: Moscow (Nauka), 181-200. (in Russian)

Gurvich, Y.G., Lukashin, V.N., Lisitzin, A.P., and Kurinov, A.D., 1980b. Rare earth elements and yttrium. In Ronov, A.B. (Ed.), Geochemistry of Hydralyzate Elements: Moscow (Nauka), 71-116. (in Russian)

Hays, J.D., et al., 1972. Init. Repts. DSDP, 9: Washington (U.S. Govt. Printing Office).

Hekinian, R., Francheteau, J., and Ballard, R.D., 1985. Morphology and evolution of hydrothermal deposits at the axis of the East Pacific Rise. Oceanol. Acta, 8:147-155.

Ivanov, G.V., and Krasnyuk., A.D., 1989. Analytical complex SPARK-1 on the base of microcomputer. 2nd All-Union Conference on XR-spectral Analy. sis, 49. (Abstract) (in Russian)

Jarvis, I., 1985. Geochemistry and origin of Eocene-Oligocene metalliferous sediments from the central equatorial Pacific: Deep Sea Drilling Project Sites 573 and 574. In Mayer, L., Theyer, F., Thomas, E., et al., Init. Repts. DSDP, 85: Washington (U.S. Govt. Printing Office), 781-804.

Krasnov, S.G., German, N.E., and Cherkashev, G.A., 1992. Distribution and main factors controlling the composition of metalliferous sediments. In Grabmerg, I.S., and Ainemer, A.I. (Eds.), Hydrothermal Sulfide Ores and Metalliferous Sediments of the Ocean: St. Petersburg (Nedra), 129-138. (in Russian)

Krasnyuk, A.D., and Ivanov, G.V., 1990. The method of analysis of oceanic sediments chemical composition by the technique of standard-background with automated spectrometer SPARK-1. Geol. Oceans Seas, 1:134. (Abstract) (in Russian)

Leinen, M., 1977. A normative calculation technique for determining opal in deep-sea sediments. Geochim. Cosmochim. Acta, 41:671-676.

Leinen, M., and Stakes, D., 1979. Metal accumulation rates in the central equatorial Pacific during Cenozoic time. Geol. Soc. Am. Bull., 90:357-375.

Lisitzin, A.P., Bogdanov, Y.A., and Gurvich, E.G., 1990. Hydrothermal Deposits of Oceanic Rift Zones: Moscow (Nauka). (in Russian)

Lonsdale, P., 1976. Abyssal circulation of the southeastern Pacific and some geological implications. J. Geophys. Res., 81:1163-1176.

Lyle, M., Leinen, M., Owen, R.M., and Rea, D.K., 1987. Late Tertiary history of hydrothermal deposition at the East Pacific Rise: correlation to volcanotectonic events. Geophys. Res. Lett., 14:595-598. 
Lyle, M., Owen, R.M., and Leinen, M., 1986. History of hydrothermal sedimentation at the East Pacific Rise, $19^{\circ} \mathrm{S}$. In Leinen, M., Rea, D.K., et al. Init. Repts. DSDP, 92: Washington (U.S. Govt. Printing Office), 585-596.

Lyutzarev, S.V., 1986. Determination of organic carbon in marine bottom sediments by the method of dry combustion. Oceanology, 4:704-708. (in Russian)

Mammerickx, J., Herron, E., and Dorman, L., 1980. Evidence for two fossil spreading ridges in the southeast Pacific. Geol. Soc. Am. Bull., 91:263271 .

Mammerickx, J., and Klitgord, K.D., 1982. Northern East Pacific Rise evolution from 25 m.y.B.P. to the present. J. Geophys. Res., 87:6751-6759.

Martin, J.M., and Knauer, G., 1973. The elemental composition of plankton. Geochim. Cosmochim. Acta, 37:1639-1653.

Mayer, L., Pisias, N., Janecek, T., et al., 1992. Proc. ODP, Init. Repts., 138 (Pts. 1 and 2): College Station, TX (Ocean Drilling Program).

Migdisov, A.A., Bogdanov, Y.A., Lisitzin, A.P., Gurvich, E.G., Lebedev, A.I., Lukashin, V.N., Gordeev, V.V., Girin, Y.P., and Sokolova, E.G., 1979. Geochemistry of metalliferous sediments. In Smirnov, V.I. (Ed.), Metalliferous Sediments of the Southeastern Pacific: Moscow (Nauka), 122-200. (in Russian)

Ponomarev, A.I., 1961. Methods of Chemical Analysis of Silicate and Carbonate Rocks: Moscow (Gostekhizdat). (in Russian)

Rea, D.K., and Leinen, M., 1986. Neogene controls on hydrothermal activity and paleoceanography of the southeast Pacific Ocean. In Leinen, M., Rea, D.K., et al., Init. Repts. DSDP, 92: Washington (U.S. Govt. Printing Office), 597-617.
Rich, J.E., Johnson, G.L., Jones, J.E., and Campsie, J., 1986. A significant correlation between fluctuations in seafloor spreading rates and evolutionary pulsations. Paleoceanography, 1:85-95.

Rosendahl, B.R., Hekinian, R., et al., 1980. Init. Repts. DSDP, 54: Washington (U.S. Govt. Printing Office).

Schrader, E.L., Furbish, W.J., Mattey, D., and May, J.A., 1980. Geochemistry and carbonate petrology of selected sediment samples from Deep Sea Drilling Project Leg 54, Eastern Pacific. In Rosendahl, B.R., Hekinian, R., et al., Init. Repts. DSDP, 54: Washington (U.S. Govt. Printing Office), 319-328.

Strakhov, N.M., 1976. Problems of Geochemistry of Recent Oceanic Lithogenesis: Moscow (Nauka). (in Russian)

Turanskaya, N.V., and Kuzmina, T.G., 1978. The method of XR-spectral fluorescent analysis in application to bottom sediments. Oceanology, 6: 1110-1114. (in Russian)

Turanskaya, N.V., Kuzmina, T.G., and Maslov, V.Y., 1976. The method of XR-spectral fluorescent analysis of silicate rocks. Oceanology, 4:713-716. (in Russian)

Turekian, K.K., and Wedepohl, K.H., 1961. Distribution of the elements in some major units of the earth's crust. Geol. Soc. Am. Bull., 72:175-192.

Date of initial receipt: 5 May 1993

Date of acceptance: 2 June 1994

Ms 138SR-147 